\title{
On the concrete representation of discrete enriched abstract clones
}

\author{
Marcelo Fiore \\ Department of Computer Science and Technology, University of Cambridge \\ E-mail: Marcelo.Fiore@cl.cam.ac.uk
}

Dedicated to Peter Freyd and Bill Lawvere.

\begin{abstract}
We consider discrete enriched abstract clones and provide two constructions investigating their representation as discrete enriched clones of operations on objects in concrete enriched categories over the enriching category. Our first construction embeds a discrete enriched abstract clone into the concrete discrete enriched clone of operations on an object in the enriching category. Our second construction refines the given embedding by introducing a monoid action and restricting attention to the concrete discrete enriched clone of its equivariant operations. As in the classical theory of abstract clones, our main focus is on discrete enriched abstract clones with finite arities. However, we also consider discrete enriched abstract clones with countable arities to show that the representation theory of the former is conceptually explained by that of the latter.
\end{abstract}

2010 Mathematics Subject Classification. 08A62. 08A65, 18C10

Keywords. Concrete and abstract clones, Lawvere theory, representation theory, finitary and infinitary algebras, enriched category theory.

\section{Introduction}

We are concerned with research themes pioneered by Bill Lawvere and Peter Freyd in the categorical theories of algebra [9, 6], representation [7], and enrichment [10]; specifically investigated here in connection with the theory of abstract clones.

The multi-sorted equational theory of abstract clones AbC, introduced by Philip Hall (see, for instance, Cohn [3, Section III.3]), is sorted by the set of natural numbers $\mathbb{N}$ and has operators

$$
\mathrm{v}_{i}^{n}: n(0 \leq i<n \in \mathbb{N}), \quad \mathbf{s}_{m, n}: m, \underbrace{n, \ldots, n}_{m \text { times }} \rightarrow n(m, n \in \mathbb{N})
$$

that respectively axiomatize the notions of variable (or projection) and substitution (or composition). An algebra (or model) of AbC

$$
C=\left\{C_{n} \in \mathcal{S} e t\right\}_{n \in \mathbb{N}} \quad, \quad \nu=\left\{\nu_{i}^{n} \in C_{n}\right\}_{0 \leq i<n \in \mathbb{N}} \quad, \quad \varsigma=\left\{\varsigma_{m, n}: C_{m} \times C_{n}{ }^{m} \longrightarrow C_{n}\right\}_{m, n \in \mathbb{N}}
$$

in the category $\mathcal{S}$ et of sets and functions is called an abstract clone. A main class of examples arises from every object $X$ that admits finite powers $\pi_{i}^{n}: X^{n} \rightarrow X(0 \leq i<n \in \mathbb{N})$ in a category $\mathscr{C}$ : the clone of operations $\mathscr{C}\langle X\rangle$ has structure given by

$$
\begin{gathered}
\left\{\mathscr{C}\left(X^{n}, X\right) \in \mathcal{S e t}\right\}_{n \in \mathbb{N}}, \quad\left\{\pi_{i}^{n}: X^{n} \rightarrow X\right\}_{0 \leq i<n \in \mathbb{N}}, \\
\left\{\mathscr{C}\left(X^{m}, X\right) \times \mathscr{C}\left(X^{n}, X\right)^{m} \cong \mathscr{C}\left(X^{m}, X\right) \times \mathscr{C}\left(X^{n}, X^{m}\right) \stackrel{{ }^{{ }^{n}}, X^{m}, X}{\longrightarrow} \mathscr{C}\left(X^{n}, X\right)\right\}_{m, n \in \mathbb{N}} .
\end{gathered}
$$


Every abstract clone can be represented as a clone of operations. This is a consequence of the deeper fact that there is an isomorphism between the category of abstract clones and the category of Lawvere theories. Recall that a Lawvere theory consists of an underlying category $\mathcal{T}$ together with an identity-on-objects strict-cartesian functor $\mathbb{F}^{\text {op }} \rightarrow \mathcal{T}$, for $\mathbb{F}$ the category of finite cardinals $([n]=\{i \in \mathbb{N} \mid i<n\}, n \in \mathbb{N})$ and functions between them equipped with its standard strict-cocartesian structure. The abstract clone associated to a Lawvere theory with underlying category $\mathcal{T}$ is the clone of operations $\mathcal{T}\langle[1]\rangle$. Conversely, an abstract clone $(C, \nu, \varsigma)$ corresponds to the Lawvere theory with underlying category $\mathcal{T}_{C}$ given by

$$
\begin{gathered}
\text { hom-sets: } \mathcal{T}_{C}([m],[n])=C_{m}{ }^{n}(m, n \in \mathbb{N}) \quad, \quad \text { identities: } \nu^{n}=\left(\nu_{i}^{n}\right)_{0 \leq i<n} \in C_{n}{ }^{n}(n \in \mathbb{N}), \\
\text { composition: } C_{m}{ }^{\ell} \times C_{n}{ }^{m} \stackrel{\left\langle\pi_{i}^{\ell} \times C_{n}{ }^{m}\right\rangle_{0 \leq i<\ell}}{\longrightarrow}\left(C_{m} \times C_{n}{ }^{m}\right)^{\ell} \stackrel{\varsigma_{m, n}{ }^{\ell}}{\longrightarrow} C_{n}{ }^{\ell} .
\end{gathered}
$$

Every abstract clone $C$ is then isomorphic to the clone of operations $\mathcal{T}_{C}\langle[1]\rangle$. There are, however, more concrete such representations.

The original notion of clone (or closed set) of operators used in universal algebra was given as a subclone of the clone of operations on a set. These clones typically arise as concrete clones of operations, by which we shall mean clones of operations in concrete categories with finite powers. A concrete category with finite powers is a category with finite powers equipped with a faithful functor to the category of sets that preserves them. Main examples are categories of algebras equipped with their forgetful functor. The clone of operations $\mathscr{A}\langle A\rangle$ on an object $A$ in a concrete category with finite powers $(\mathscr{A}, U: \mathscr{A} \longrightarrow \mathcal{S}$ et $)$ is then a subclone of the clone of operations $\mathcal{S} e t\langle U A\rangle$ on the set $U A$ by means of the injections

$$
\mathscr{A}\langle A\rangle_{n}=\mathscr{A}\left(A^{n}, A\right) \stackrel{U_{A^{n}, A}}{\longrightarrow} \operatorname{Set}\left(U\left(A^{n}\right), U A\right) \cong \operatorname{Set}\left((U A)^{n}, U A\right) .
$$

The question arises as to whether every abstract clone may be represented as a concrete clone of operations. To discuss the answer we need address a technicality of the theory of abstract clones.

The literature on universal algebra often considers two different notions of abstract clone: the one given above, which in virtue of its correspondence with Lawvere theories we regard as fundamental, and a restricted one arising from the multi-sorted equational theory of positive abstract clones $\mathrm{AbC}^{+}$ defined as the restriction of $A b C$ to the set of sorts $\mathbb{N}^{+}$of positive natural numbers. Thus, the signature of $\mathrm{AbC}$ is that of $\mathrm{AbC}^{+}$extended with the sort 0 and the operators $\mathrm{s}_{0, n}: 0 \rightarrow n(n \in \mathbb{N})$. From the perspective of Lawvere theories, positive abstract clones exclude the direct consideration of nullary operators, which are typically justified indirectly as constant unary operators. This amounts, from the perspective of mono-sorted equational presentations, to introducing constants e as unary operators subject to the equation $\mathrm{e}(x)=\mathrm{e}(y)$ ( $c f$. the early categorical axiomatization of the theory of groups by Peter Freyd in [6]).

Coming back to the concrete representation of abstract clones, it is a folklore theorem of the theory of abstract clones, for which see e.g. Sangalli [17] and Taylor [20, Lecture III] that further refer to $[16,18,19]$, that every positive abstract clone is isomorphic to a positive clone of operators in a category of unary algebras. It is this result that we investigate here for suitable enriching categories other than $\mathcal{S}$ et. The need for restricting attention to positive abstract clones will become apparent in our development.

Let us discuss the categorical setting in which we shall be working. As in the classical theory of abstract clones, we wish to consider abstract clones with discrete finite arities enriched in a 
category $\mathcal{S}$, henceforth referred to as df-abstract $\mathcal{S}$-clones, and also to internalize the clone of discrete finitary operations on an object $S$ in $\mathcal{S}$ as a discrete finitary $\mathcal{S}$-clone of operations $\underline{\mathcal{S}}\langle S\rangle$. The former requires $\mathcal{S}$ to be monoidal with finite powers, while the latter that it also be closed. This is the minimal categorical structure needed on the enriching category. In this setting, as a stepping stone to the concrete-representation problem, we shall consider the following.

Embedding problem. For a monoidal closed category with finite powers $\mathcal{S}$ and a df-abstract $\mathcal{S}$-clone $C$, construct an object $\underline{C}$ in $\mathcal{S}$ and an embedding from the df-abstract $\mathcal{S}$-clone $C$ to the concrete discrete finitary $\mathcal{S}$-clone of operations $\underline{\mathcal{S}}\langle\underline{C}\rangle$.

As we will show elsewhere, the embedding problem has no solution for the topos of finite sets and functions. Infinitary structure is thus necessary and, in Section 3, we present a solution to the embedding problem for biclosed enriching categories with colimits of $\omega$-chains of sections that are preserved by finite powers.

Next we investigate concrete representations. We define a concrete cartesian $\mathcal{S}$-category with finite powers $\mathscr{A}$ to be an $\mathcal{S}$-category with finite powers together with a faithful $\mathcal{S}$-functor $\underline{\mathscr{A}} \rightarrow \underline{\mathcal{S}}$ that preserves finite powers, and in this context consider the following.

Concrete-representation problem. For a monoidal closed category with finite powers $\mathcal{S}$ and a df-abstract $\mathcal{S}$-clone $C$, construct a concrete $\mathcal{S}$-category with finite powers $\mathscr{A}$ and an object $A$ in it together with an isomorphism from the df-abstract $\mathcal{S}$-clone $C$ to the concrete discrete finitary $\mathcal{S}$-clone of operations $\mathscr{A}\langle A\rangle$ on $A$.

In Section 4, building upon the embedding construction of Section 3 and in the vein of the aforementioned concrete representation of abstract clones in universal algebra, we present an actionrepresentation construction addressing the following.

Action-representation problem. For a monoidal closed category with finite powers and equalizers $\mathcal{S}$ and a df-abstract $\mathcal{S}$-clone $C$, construct a monoid $\mathrm{M}(C)$ and an $\mathrm{M}(C)$-action $\mathrm{A}(C)$ in $\mathcal{S}$ together with an isomorphism from the df-abstract $\mathcal{S}$-clone $C$ to the discrete finitary $\mathcal{S}$-clone of operations $\underline{\mathcal{S}}^{\mathrm{M}(C)}\langle\mathrm{A}(C)\rangle$ on $\mathrm{A}(C)$ in the concrete $\mathcal{S}$-category with finite powers $\underline{\mathcal{S}}^{\mathrm{M}(C)}$ of $\mathrm{M}(C)$-actions.

Specifically, we solve this problem for positive df-abstract $\mathcal{S}$-clones under the further assumptions on the enriching category $\mathcal{S}$ that it is biclosed, has colimits of $\omega$-chains of sections that are preserved by finite powers, and also has countable powers.

Section 5 develops a conceptual framework for the embedding and representation theorems of the previous two sections. This necessitates the consideration of enriched clones with operators of discrete countable arity, henceforth referred to as dc-abstract enriched clones. For them, Cayleystyle embedding and representation theorems are established, and it is shown that the representation of a df-abstract enriched clone arises from that of the free dc-abstract enriched clone on it.

The investigation of the category-theoretic enrichment of universal algebra (see e.g. [4, 2, 14, $15,13,8,11]$ ) has lead to several variations and generalizations of the notion of Lawvere theory. In particular, Dubuc [4] introduced enriched theories with arbitrary arities; Borceux and Day [2] considered enriched theories with discrete finite arities; Power [14] generalized the classical category-theoretic approach to universal algebra by means of enriched Lawvere theories with finitely presentable arities; Power [15] studied discrete countable enriched Lawvere theories; and 
Lucyshyn-Wright [11] introduced a general notion of enriched theory with respect to a system of arities. As for an associated notion of enriched abstract clone, we are not aware of it being made explicit and, in this respect, present the following.

Definition 1.1. Let $(\mathcal{S}, I, \otimes)$ be a monoidal category and let $A=\{\widehat{a} \in \mathcal{S}\}_{a \in \mathcal{A}}$ be an indexed family of objects such that $\mathcal{S}$ has powers $[\widehat{a},-]$ for all $a \in \mathcal{A}$.

An $A$-abstract $\underline{\mathcal{S}}$-clone $(Z, \nu, \varsigma)$ is a structure

$$
\begin{gathered}
Z=\left\{Z_{a} \in \mathcal{S}\right\}_{a \in \mathcal{A}}, \nu=\left\{\nu^{a}: I \longrightarrow\left[\widehat{a}, Z_{a}\right] \text { in } \mathcal{S}\right\}_{a \in \mathcal{A}}, \\
\varsigma=\left\{\varsigma_{a, b}: Z_{a} \otimes\left[\widehat{a}, Z_{b}\right] \rightarrow Z_{b} \text { in } \mathcal{S}\right\}_{a, b \in \mathcal{A}}
\end{gathered}
$$

satisfying the following laws

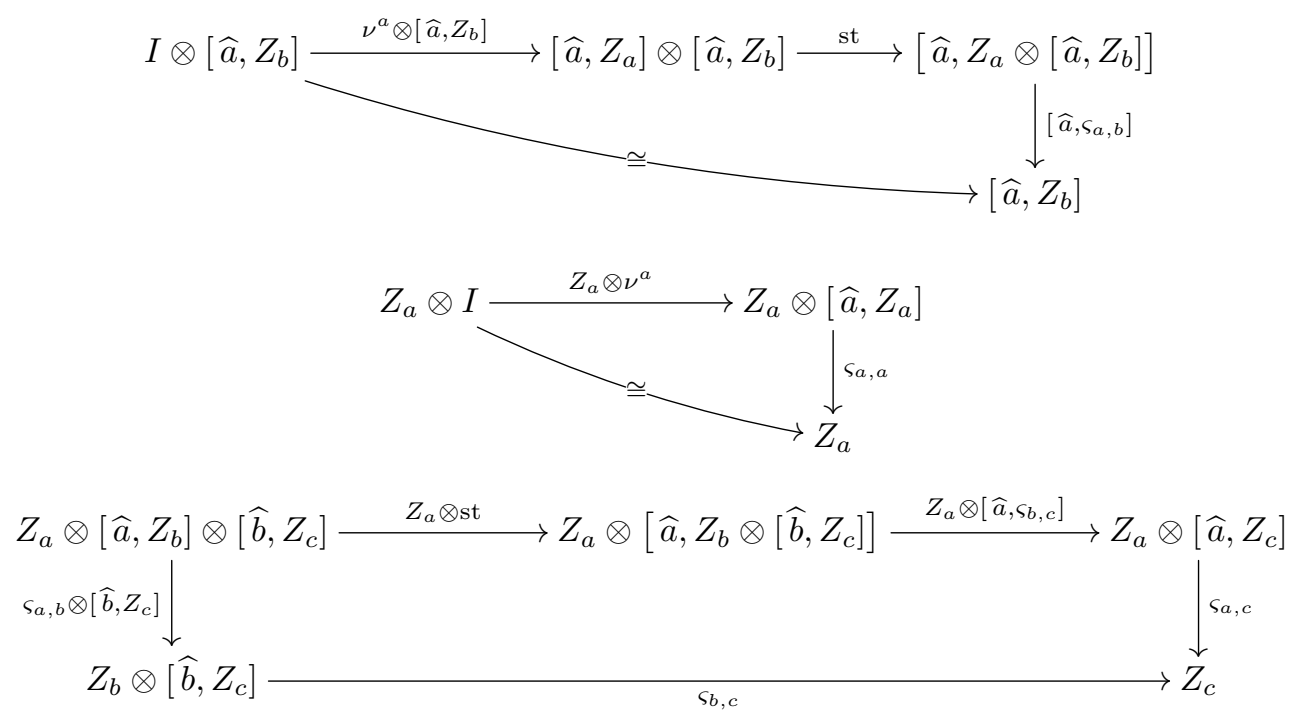

where st $X, Y, Z$ stands for the standard tensorial strength $[X, Y] \otimes Z \rightarrow[X, Y \otimes Z]$.

Remark 1.2. Enriched abstract clones may be seen in the context of the enriched version of relative monads over a functor, introduced in the unenriched setting in [1] and considered in the bicategorical setting in [5]. Indeed, in the setting of enrichment with respect to a monoidal left closed category $\mathcal{S}$, an $\underline{A}$-relative $\mathcal{S}$-monad for an $\mathcal{S}$-functor $\underline{A}: \mathcal{A} \rightarrow \mathcal{S}$ is essentially an $A$-abstract $\mathcal{S}$-clone for the indexed family $A$ determined by the object mapping of $\underline{A}$.

The notion of $A$-abstract $\mathcal{S}$-clone essentially corresponds to: $(i)$ Dubuc's enriched theories [4] for $A$ the objects of $\mathcal{S}$; (ii) Borceux and Day's enriched theories [2] for $A$ the finite coproducts of the unit of $\mathcal{S}$; (iii) Power's enriched Lawvere theories [14] for $A$ the finitely presentable objects of $\mathcal{S}$; $(i v)$ Power's discrete countable Lawvere theories [15] for $A$ the countable coproducts of the unit 
of $\mathcal{S}$; and $(v)$ Lucyshyn-Wright's enriched algebraic theories with arities [11] for $A$ the system of arities.

Our work provides concrete representations of discrete finitary and discrete countable enriched abstract clones, respectively corresponding to Borceux and Day's enriched theories [2] and to Power's discrete countable enriched Lawvere theories [15]. It would be interesting to extend our results to a wider class of enriched abstract clones also encompassing non-discrete arities.

\section{Enriched abstract clones with discrete finite arities}

This section introduces the necessary background of the paper. We provide basic definitions and examples of enriched abstract clones with discrete finite arities, and make explicit some of their structure.

The classical concept of abstract clone can be enriched in monoidal categories with finite powers. We therefore adopt the following situation.

Assumption 2.1. Henceforth, we work with an enriching monoidal category $\mathcal{S}$ (with unit and tensor product respectively denoted by $I$ and $\otimes$ ) with finite powers (denoted $(-)^{n}$ for $n \in \mathbb{N}$ ).

Notation 2.2. Recall that, for $n \in \mathbb{N}$, we write $[n]$ for the finite cardinal $\{i \in \mathbb{N} \mid i<n\}$.

Definition 2.3 (Discrete finitary enriched abstract clones). A df-abstract $\mathcal{S}$-clone $(C, \nu, \varsigma)$ is a structure

$$
\begin{gathered}
C=\left\{C_{n} \in \mathcal{S}\right\}_{n \in \mathbb{N}}, \nu=\left\{\left\langle\nu_{i}^{n}\right\rangle_{i \in[n]}: I \longrightarrow C_{n}{ }^{n} \text { in } \mathcal{S}\right\}_{n \in \mathbb{N}}, \\
\varsigma=\left\{\varsigma_{m, n}: C_{m} \otimes C_{n}{ }^{m} \longrightarrow C_{n} \text { in } \mathcal{S}\right\}_{m, n \in \mathbb{N}}
\end{gathered}
$$

satisfying the following laws

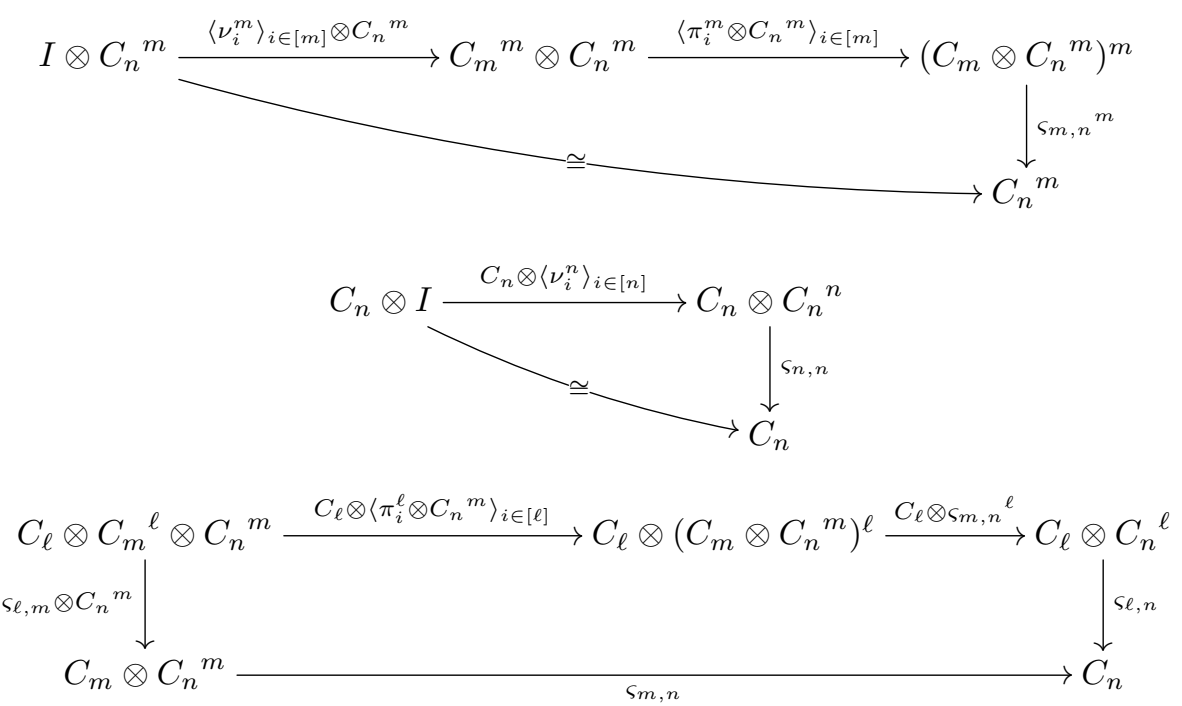


Remark 2.4. The notion of discrete finitary enriched abstract clone above is essentially a rewording of Borceux and Day's notion of enriched theory (see [2, Definition 2.1.1], and also consult [11]) in the language of abstract clones.

For a df-abstract $\mathcal{S}$-clone $(C, \nu, \varsigma)$, one typically refers to $C$ as the carrier and to the structures $\nu$ and $\varsigma$ respectively as the variables and substitution.

Main examples of df-abstract $\mathcal{S}$-clones are given by clones of operations on objects in $\mathcal{S}$-categories with finite powers. To introduce them, let us recall the notion of finite product in the enriched setting. The product of $\left\{A_{i}\right\}_{i \in[n]}(n \in \mathbb{N})$ in an $\mathcal{S}$-category $\mathscr{A}$ is specified by isomorphisms

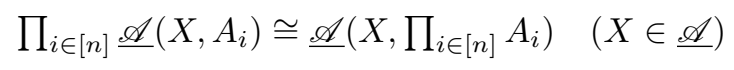

that are $\mathcal{S}$-natural.

Example 2.5 (Discrete finitary enriched clone of operations). Let $\mathscr{A}$ be an $\mathcal{S}$-category with finite powers. The discrete finitary $\mathcal{S}$-clone of operations $\mathscr{A}\langle X\rangle$ on an object $X \in \underline{A}$ is given by the family $\underline{\mathscr{A}}\langle X\rangle_{n}=\underline{\mathscr{A}}\left(X^{n}, X\right)(n \in \mathbb{N})$ with variables given by the composite

$$
I \stackrel{\operatorname{id}_{X}{ }^{n}}{\longrightarrow} \mathscr{A}\left(X^{n}, X^{n}\right) \cong \mathscr{A}\left(X^{n}, X\right)^{n} \stackrel{\pi_{i}^{n}}{\longrightarrow} \underline{\mathscr{A}}\left(X^{n}, X\right) \quad(i \in[n])
$$

and substitution by the composite

$$
\underline{\mathscr{A}}\left(X^{m}, X\right) \otimes \underline{\mathscr{A}}\left(X^{n}, X\right)^{m} \cong \underline{\mathscr{A}}\left(X^{m}, X\right) \otimes \underline{\mathscr{A}}\left(X^{n}, X^{m}\right) \stackrel{{ }^{X^{n}, X^{m}, X}}{\longrightarrow} \underline{\mathscr{A}}\left(X^{n}, X\right) .
$$

The rest of the section identifies functorial structure that is implicit in the algebraic structure of df-abstract clones and is important to reveal further structure imposed by variables and substitution on the carrier.

Notation 2.6. Recall that we write $\mathbb{F}$ for the category of finite cardinals and functions.

Definition 2.7. Let $C$ be a df-abstract $\mathcal{S}$-clone. For $n, m \in \mathbb{N}$ and $f:[n] \longrightarrow[m]$ in $\mathbb{F}$, define the action $C_{f}: C_{n} \rightarrow C_{m}$ in $\mathcal{S}$ as the composite

$$
C_{n} \cong C_{n} \otimes I \stackrel{C_{n} \otimes\left\langle\nu_{f i}^{m}\right\rangle_{i \in[n]}}{\longrightarrow} C_{n} \otimes C_{m}{ }^{n} \stackrel{\varsigma_{n, m}}{\longrightarrow} C_{m}
$$

Proposition 2.8. Let $C$ be a df-abstract $\mathcal{S}$-clone. For all $n, m, \ell \in \mathbb{N}$, and $[n] \stackrel{f}{\rightarrow}[m] \stackrel{g}{\longrightarrow}[\ell]$ in $\mathbb{F}$, we have the following identities.

$$
\begin{gathered}
C_{\mathrm{id}_{[n]}}=\operatorname{id}_{C_{n}}, \quad C_{(g f)}=C_{g} C_{f}: C_{n} \rightarrow C_{\ell} \\
\underbrace{C_{C_{m}}^{\nu_{i}^{n}}}_{\nu_{f i}^{m}} \underbrace{C_{n}}_{C_{f}}(i \in[n])
\end{gathered}
$$



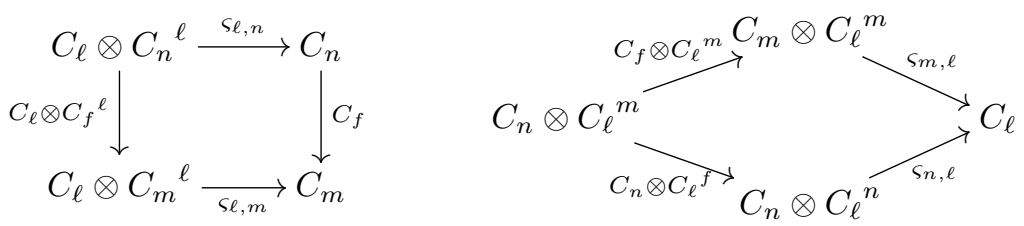

The corollary below recasts (1) and (2) above in categorical form.

Corollary 2.9. Let $C$ be a df-abstract $\mathcal{S}$-clone.

1. The mappings

$$
[n] \in \mathbb{F} \mapsto C_{n} \in \mathcal{S}(n \in \mathbb{N}), f:[n] \longrightarrow[m] \text { in } \mathbb{F} \mapsto C_{f}: C_{n} \longrightarrow C_{m} \text { in } \mathcal{S}
$$

define a functor $C: \mathbb{F} \longrightarrow \mathcal{S}$.

2. For all $\ell \in \mathbb{N}$, the family

$$
\varsigma_{\ell,-}=\left\{\varsigma_{\ell, k}: C_{\ell} \otimes C_{k}^{\ell} \rightarrow C_{k}\right\}_{k \in \mathbb{F}}
$$

defines a natural transformation $C_{\ell} \otimes C_{(-)}^{\ell} \stackrel{\cdot}{\rightarrow} C_{(-)}: \mathbb{F} \rightarrow \mathcal{S}$.

3. For all $\ell \in \mathbb{N}$, the family

$$
\varsigma_{-, \ell}=\left\{\varsigma_{k, \ell}: C_{k} \otimes C_{\ell}{ }^{k} \rightarrow C_{\ell}\right\}_{k \in \mathbb{F}}
$$

defines a dinatural transformation $C_{(=)} \otimes C_{\ell}(-) \stackrel{\cdot}{\rightarrow} C_{\ell}: \mathbb{F}^{\mathrm{op}} \times \mathbb{F} \rightarrow \mathcal{S}$.

Homomorphisms of df-abstract enriched clones are structure-preserving maps between their underlying carriers. We record the definition and note that they are natural with respect to the functorial action of df-abstract enriched clones.

Definition 2.10. An homomorphism $h:(C, \nu, \varsigma) \longrightarrow\left(C^{\prime}, \nu^{\prime}, \varsigma^{\prime}\right)$ of df-abstract $\mathcal{S}$-clones is a family

$$
h_{n}: C_{n} \rightarrow C_{n}^{\prime} \text { in } \mathcal{S}(n \in \mathbb{N})
$$

such that the diagrams
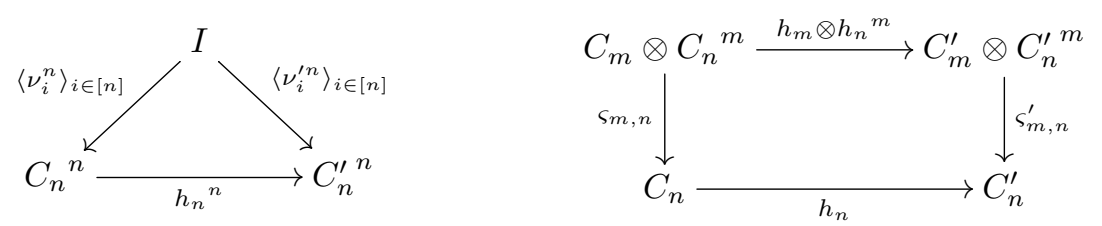

commute for all $m, n \in \mathbb{N}$.

Definition 2.11. For a monoidal category with finite powers $\mathcal{S}$, we let $\mathcal{A} b \mathcal{C}(\mathcal{S})$ be the category of df-abstract $\mathcal{S}$-clones and their homomorphisms.

Proposition 2.12. An homomorphism $C \longrightarrow C^{\prime}$ of df-abstract $\mathcal{S}$-clones $C$ and $C^{\prime}$ yields a natural transformation $C \rightarrow C^{\prime}: \mathbb{F} \longrightarrow \mathcal{S}$.

Definition 2.13. A (positive) embedding of df-abstract enriched clones is an homomorphism for which every (positive) component is a monomorphism. 


\subsection{Nullary operations as multi-ary ones}

Every injection with non-empty domain in $\mathbb{F}$ is a section and hence preserved by the functor $C$ : $\mathbb{F} \rightarrow \mathcal{S}$ induced by a df-abstract $\mathcal{S}$-clone $C$. We proceed to provide conditions under which the action $\left.C_{([0]} \hookrightarrow[n]\right): C_{0} \rightarrow C_{n}(n \in \mathbb{N})$ associated to the inclusion $[0] \hookrightarrow[n]$ in $\mathbb{F}$ is also monomorphic. For this and subsequent developments, the following definition will be convenient.

Definition 2.14. For every pair $k, \ell \in \mathbb{N}$ with $k=0$ whenever $\ell=0$, we let $k \rightarrow \ell$ denote the function $[k] \longrightarrow[\ell]$ in $\mathbb{F}$ given by the mapping $i \in[k] \mapsto \min (i, \ell-1) \in[\ell]$.

Inspecting the definition, one sees that, for $k \leq \ell \in \mathbb{N}$, the function $k \rightarrow \ell$ is the inclusion $[k] \hookrightarrow[\ell]$, while the function $\ell \rightarrow k$ is its unique order-preserving retraction.

\subsubsection{Monoidal enrichment}

When the object of nullary operations is inhabited, it is a section of the multi-ary ones.

Definition 2.15. An object $X$ in a monoidal category with unit $I$ is said to be inhabited whenever it has a generalized element of the form $I \rightarrow X$.

Proposition 2.16. For every df-abstract $\mathcal{S}$-clone $C$ with $C_{0}$ inhabited and $n \in \mathbb{N}$, the action $C_{(0 \rightarrow n)}$ : $C_{0} \rightarrow C_{n}$ is a section.

Proof. Let $C$ be a df-abstract $\mathcal{S}$-clone with substitution structure $\varsigma$. For every generalized element $c: I \rightarrow C_{0}$ and $n \in \mathbb{N}$, we have the following commutative diagram

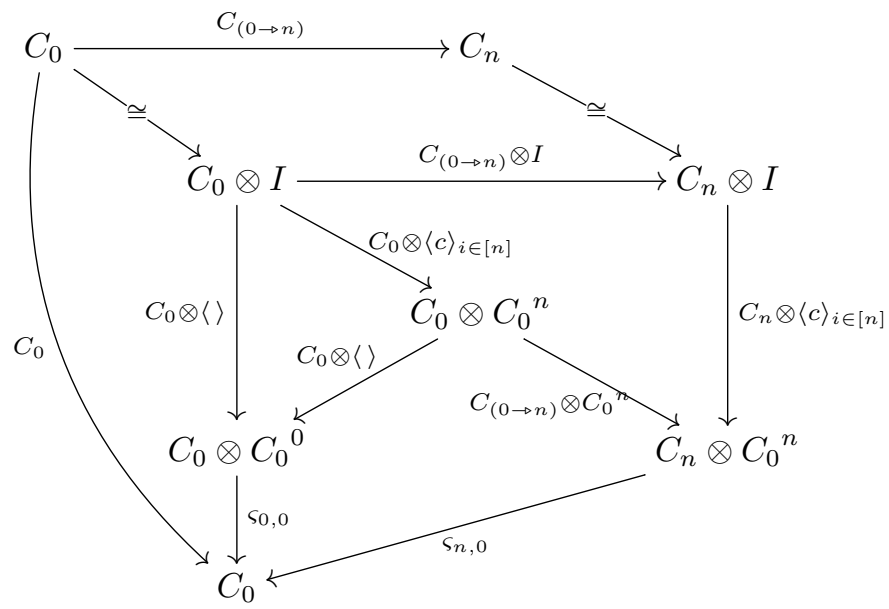

Q.E.D.

Corollary 2.17. The functor $C: \mathbb{F} \rightarrow \mathcal{S}$ induced by a df-abstract $\mathcal{S}$-clone $C$ with $C_{0}$ inhabited preserves monomorphisms.

\subsubsection{Cartesian enrichment}

Assumption 2.18. For the rest of this section, we restrict attention to the case in which the enriching category $\mathcal{S}$ is cartesian monoidal. 
In the cartesian enriched setting, for a df-abstract $\mathcal{S}$-clone $C$, the action $C_{(0 \rightarrow n)}(n \in \mathbb{N})$ and the substitution map $\varsigma_{0, n}$ are interdefinable.

Proposition 2.19. Let $C$ be a df-abstract $\mathcal{S}$-clone. For all $n \in \mathbb{N}$, the diagram

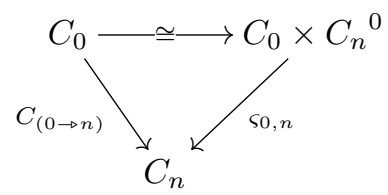

commutes.

Let us now consider the extent to which the action maps $C_{(0 \rightarrow n)}(n \in \mathbb{N})$ of a df-abstract $\mathcal{S}$-clone $C$ may be considered parametrized sections. To this end we introduce the following notion.

Definition 2.20. A map $s: X \rightarrow Y$ in a cartesian category is said to be an $n$-section, for $n \in \mathbb{N}$, if there exists $r: Y \times X^{n} \rightarrow X$ such that the diagram

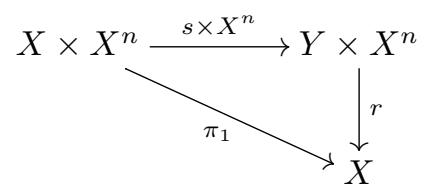

commutes.

Proposition 2.21. 1. The notions of section and 0-section are equivalent.

2. In every cartesian category, $n$-sections are monomorphisms for all $n \in \mathbb{N}$.

3. If $s: X \rightarrow Y$ is an $n$-section for some $1 \leq n \in \mathbb{N}$ and $X$ has a global section then $s$ is a section.

It follows that the insertion of nullary operators into multi-ary ones is monomorphic.

Proposition 2.22. Let $C$ be a df-abstract $\mathcal{S}$-clone. The maps $C_{(0 \rightarrow n)}: C_{0} \longrightarrow C_{n}(n \in \mathbb{N})$ are $n$-sections.

Proof. Using Proposition 2.19, one has that the diagram

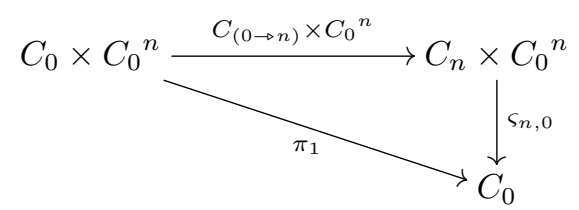

commutes.

Q.E.D.

Corollary 2.23. For a cartesian monoidal enriching category $\mathcal{S}$, the functor $\mathbb{F} \longrightarrow \mathcal{S}$ induced by a df-abstract $\mathcal{S}$-clone preserves monomorphisms.

Proof. Follows from Proposition 2.22 and the fact that every monomorphism with non-empty domain in $\mathbb{F}$ is a section.

Q.E.D. 


\section{Embedding theorem}

The aim of this section is to give a solution to the embedding problem presented in the introduction. As mentioned there, besides being monoidal (right) closed and having finite powers, the enriching category need support further structure. We will motivate the structure we will be requiring by explaining the basic idea behind our construction.

Assumption 3.1. Henceforth, we work with an enriching monoidal closed category $\mathcal{S}$ with finite powers.

Notation 3.2. The category $\mathcal{S}$ enriched over itself will be denoted $\underline{\mathcal{S}}$. Accordingly, $\underline{\mathcal{S}}(X, Y) \in \mathcal{S}$ is the internal hom from $X$ to $Y$ in $\mathcal{S}$, and we write $\varepsilon_{X, Y}: \underline{\mathcal{S}}(X, Y) \otimes X \rightarrow Y$ for its evaluation map.

In view of the following proposition, one may consider discrete finitary $\mathcal{S}$-clones of operations on objects of $\underline{\mathcal{S}}$.

Proposition 3.3. The $\mathcal{S}$-category $\underline{\mathcal{S}}$ has finite powers.

A unitary Menger algebra of rank $n \in \mathbb{N}$ (see e.g. $[12,18]$ for this notion) is a model of the equational theory of abstract clones restricted to the single sort $n$, with operators

$$
\mathrm{v}_{i}^{n}: n(i \in[n]), \quad \mathrm{s}_{n, n}: n, \underbrace{n, \ldots, n}_{n \text { times }} \rightarrow n .
$$

Every df-abstract $\mathcal{S}$-clone $(C, \nu, \varsigma)$ induces two unitary Menger algebras of rank $n \in \mathbb{N}$ in $\mathcal{S}$ :

$$
\begin{gathered}
C_{n} \in \mathcal{S}, \quad\left\langle\nu_{i}^{n}\right\rangle_{i \in[n]}: I \rightarrow C_{n}{ }^{n} \text { in } \mathcal{S}, \\
\varsigma_{n, n}: C_{n} \otimes C_{n}{ }^{n} \rightarrow C_{n} \text { in } \mathcal{S}
\end{gathered}
$$

and

$$
\begin{array}{r}
\underline{\mathcal{S}}\left(C_{n}{ }^{n}, C_{n}\right) \in \mathcal{S}, \quad I \rightarrow \underline{\mathcal{S}}\left(C_{n}{ }^{n}, C_{n}\right)^{n} \text { in } \mathcal{S}, \\
\quad \underline{\mathcal{S}}\left(C_{n}{ }^{n}, C_{n}\right) \otimes \underline{\mathcal{S}}\left(C_{n}{ }^{n}, C_{n}\right)^{n} \rightarrow \underline{\mathcal{S}}\left(C_{n}{ }^{n}, C_{n}\right)
\end{array}
$$

with the former admitting a Cayley-style embedding into the latter as follows

$$
\sigma_{n}: C_{n} \longmapsto \underline{\mathcal{S}}\left(C_{n}{ }^{n}, C_{n}\right)
$$

and obtained by right transposing the substitution structure (3). We shall proceed to construct an object $\underline{C} \in \mathcal{S}$ and a positive embedding of $\mathcal{S}$-clones

$$
\rho: C \longrightarrow \underline{\mathcal{S}}\langle\underline{C}\rangle
$$

with positive components

$$
\rho_{n}: C_{n} \longrightarrow \underline{\mathcal{S}}\left(\underline{C}^{n}, \underline{C}\right)
$$


that restrict to the embeddings (4). This we will achieve by assembling all the positive $n$-ary operators

$$
C_{1} \stackrel{C_{(1 \rightarrow 2)}}{\longrightarrow} C_{2} \rightarrow \cdots \rightarrow C_{n} \stackrel{C_{(n \rightarrow n+1)}}{\longrightarrow} C_{n+1} \rightarrow \cdots \quad(1 \leq n \in \mathbb{N})
$$

by means of a colimit construction.

Assumption 3.4. Henceforth, we will work with an enriching monoidal biclosed category $\mathcal{S}$ assumed to have colimits of $\omega$-chains of sections and finite powers that preserve them.

Definition 3.5. For a df-abstract $\mathcal{S}$-clone $C$, we let $\left\{\imath_{n}: C_{n} \rightarrow \underline{C}\right\}_{1 \leq n \in \mathbb{N}}$ be a colimit of the $\omega$-chain $\left\{C_{(k \rightarrow k+1)}: C_{k} \succ C_{k+1}\right\}_{1 \leq k \in \mathbb{N}}$ and define $\imath_{0}: C_{0} \rightarrow \underline{C}$ as the composite $\imath_{1} C_{(0 \rightarrow 1)}$.

We remark that the above construction is functorial, and proceed to analyze the structure of the colimiting cone.

Proposition 3.6. The mapping $C \longmapsto \underline{C}$ extends to a functor $\mathcal{A b C}(\mathcal{S}) \rightarrow \mathcal{S}$ from the category of df-abstract $\mathcal{S}$-clones to $\mathcal{S}$.

Definition 3.7. Let $C$ be a df-abstract $\mathcal{S}$-clone. For $1 \leq n \in \mathbb{N}$, we have that the family $\left\{C_{(k \rightarrow n)}: C_{k} \longrightarrow C_{n}\right\}_{1 \leq k \in \mathbb{N}}$ is a cone for the $\omega$-chain $\left\{C_{(k \rightarrow k+1)}: C_{k} \succ C_{k+1}\right\}_{1 \leq k \in \mathbb{N}}$ and we define $\jmath_{n}: \underline{C} \longrightarrow C_{n}$ as the unique mediating map such that $\jmath_{n} \imath_{k}=C_{(k \rightarrow n)}$ for all $1 \leq k \in \mathbb{N}$.

Proposition 3.8. Let $C$ be a df-abstract $\mathcal{S}$-clone. For all $1 \leq n \in \mathbb{N}$, the map $\imath_{n}: C_{n} \rightarrow \underline{C}$ is a section with retraction $\jmath_{n}: \underline{C} \longrightarrow C_{n}$.

Having set the scene, the rest of the section establishes an embedding theorem in three steps: (i) we define a family of maps $\rho=\left\{\rho_{n}\right\}_{n \in \mathbb{N}}$ as in (5) above; (ii) we verify that $\rho$ is an $\mathcal{S}$-clone homomorphism $C \rightarrow \underline{\mathcal{S}}\langle\underline{C}\rangle$; and (iii) we show that every positive component of $\rho$ is monomorphic.

Definition 3.9. For a df-abstract $\mathcal{S}$-clone $C$ with substitution structure $\varsigma$, define

$$
\alpha=\left\{\alpha_{n}: C_{n} \otimes \underline{C}^{n} \rightarrow \underline{C}\right\}_{n \in \mathbb{N}}
$$

with components given by the composite

$$
C_{n} \otimes \underline{C}^{n} \cong \operatorname{colim}_{1 \leq m \in \mathbb{N}} C_{n} \otimes C_{m}^{n} \stackrel{\operatorname{colim}_{1 \leq m \in \mathbb{N}} \varsigma_{n, m}}{\longrightarrow} \operatorname{colim}_{1 \leq m \in \mathbb{N}} C_{m}=\underline{C} .
$$

Further, let

$$
\rho=\left\{\rho_{n}: C_{n} \rightarrow \underline{\mathcal{S}}\left(\underline{C}^{n}, \underline{C}\right)\right\}_{n \in \mathbb{N}}
$$

be given by right transposing each component of $\alpha$.

Note that the definition of $\alpha$ relies on the enriching category being left closed and on Corollary $2.9(2)$.

Proposition 3.10. For a df-abstract $\mathcal{S}$-clone $C$, the family $\alpha$ defines a dinatural transforma-

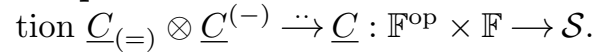


Proof. Follows from Proposition 2.9 (3).

Q.E.D.

The relationship between (4) and (5) is as follows.

Proposition 3.11. For every df-abstract $\mathcal{S}$-clone, $\rho_{0}=\underline{\mathcal{S}}\left(\langle\rangle, \imath_{0}\right) \sigma_{0}$ and, for all $1 \leq n \in \mathbb{N}$, $\sigma_{n}=\underline{\mathcal{S}}\left(\imath_{n}{ }^{n}, \jmath_{n}\right) \rho_{n}$.

Proposition 3.12. Let $(C, \nu, \varsigma)$ be a df-abstract $\mathcal{S}$-clone. The family $\rho$ is an $\mathcal{S}$-clone homomorphism $C \longrightarrow \underline{\mathcal{S}}\langle\underline{C}\rangle$.

Proof. See Appendix A.

Q.E.D.

We are left with showing that each positive component of $\rho$ is monomorphic. We require the following.

Definition 3.13. Let $C$ be a df-abstract $\mathcal{S}$-clone with variables $\nu$. For $i \in \mathbb{N}$, let $\underline{\nu}_{i}: I \rightarrow \underline{C}$ be given by the composites

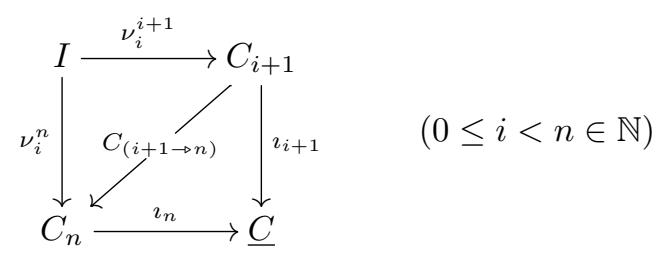

and, for $n \in \mathbb{N}$, set

$$
\eta_{n}=\left\langle\underline{\nu}_{i}\right\rangle_{i \in[n]}: I \rightarrow \underline{C}^{n} .
$$

Proposition 3.14. Let $C$ be a df-abstract $\mathcal{S}$-clone. For $n \in \mathbb{N}$,

$$
\imath_{n}=\left(C_{n} \cong C_{n} \otimes I \stackrel{C_{n} \otimes \eta_{n}}{\longrightarrow} C_{n} \otimes \underline{C}^{n} \stackrel{\alpha_{n}}{\longrightarrow} \underline{C}\right) .
$$

Proof. By the commutativity of the following diagram:

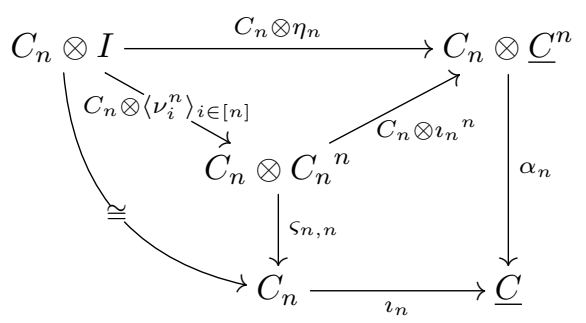

Q.E.D.

Proposition 3.15. Let $C$ be a df-abstract $\mathcal{S}$-clone. The map $\rho_{n}: C_{n} \rightarrow \underline{\mathcal{S}}\left(\underline{C}^{n}, \underline{C}\right)$ is a section for all $1 \leq n \in \mathbb{N}$. 
Proof. Follows from Propositions 3.8 and 3.14 because, for $1 \leq n \in \mathbb{N}$, the diagram

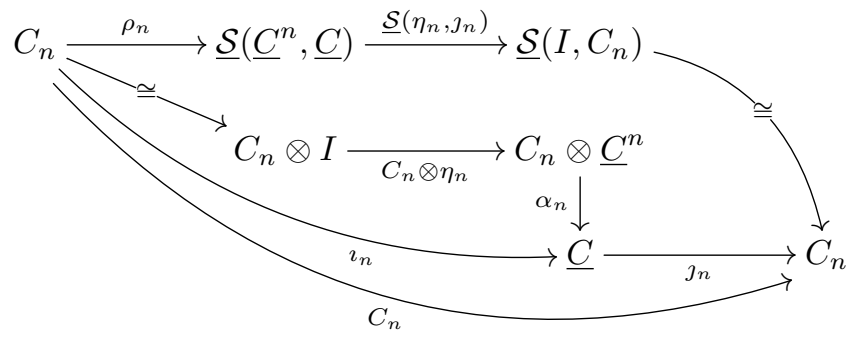

commutes.

Q.E.D.

Our development summarizes as follows.

Theorem 3.16 (Embedding theorem). Let $\mathcal{S}$ be a monoidal biclosed category with colimits of $\omega$-chains of sections and finite powers that preserve them. For every df-abstract $\mathcal{S}$-clone $C$, the family $\rho$ provides a positive embedding of $C$ into the concrete discrete finitary $\mathcal{S}$-clone of operations $\underline{\mathcal{S}}\langle\underline{C}\rangle$ on $\underline{C}$ in $\underline{\mathcal{S}}$. Moreover, this is an embedding whenever $C_{0}$ is inhabited.

Proof. The first part follows from Propositions 3.12 and 3.15. In addition, the second part uses Proposition 2.16.

Q.E.D.

\subsection{Cartesian enrichment}

Assumption 3.17. For the rest of this section, we restrict attention to the case in which the enriching category $\mathcal{S}$ is cartesian monoidal.

Proposition 3.18. Let $C$ be a df-abstract $\mathcal{S}$-clone. The map $\imath_{0}: C_{0} \rightarrow \underline{C}$ is a 1-section.

Proof. Write $\varsigma$ for the substitution structure. For $1 \leq k \in \mathbb{N}$, we have

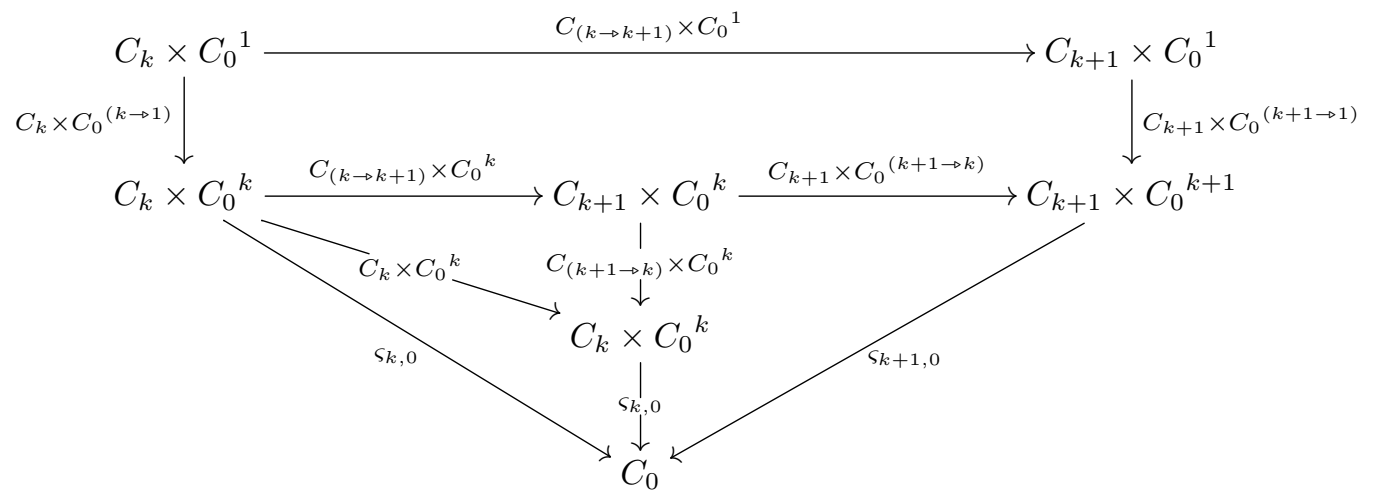

so that the family $\left\{C_{k} \times C_{0}{ }^{1} \stackrel{C_{k} \times C_{0}{ }^{(k \rightarrow 1)}}{\longrightarrow} C_{k} \times C_{0}{ }^{k} \stackrel{\varsigma_{k, 0}}{\longrightarrow} C_{0}\right\}_{1 \leq k \in \mathbb{N}}$ is a cone for the $\omega$-chain $\left\{C_{(k \rightarrow k+1)} \times C_{0}{ }^{1}: C_{k} \times C_{0}{ }^{1} \rightarrow C_{k+1} \times C_{0}{ }^{1}\right\}_{1 \leq k \in \mathbb{N}}$. Then, the unique mediating map $\jmath_{0}: \underline{C} \times$ 
$C_{0}{ }^{1} \rightarrow C_{0}$ is such that

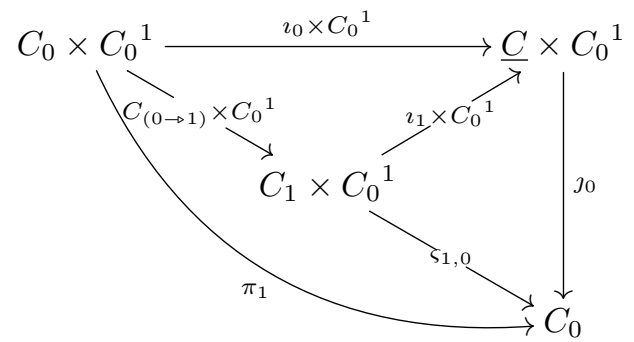

Q.E.D.

Corollary 3.19. Let $C$ be a df-abstract $\mathcal{S}$-clone. The map $\rho_{0}: C_{0} \rightarrow \underline{\mathcal{S}}\left(\underline{C^{0}}, \underline{C}\right)$ is a 1-section.

Proof. Follows from Proposition 3.18, because the composite $C_{0} \stackrel{\rho_{0}}{\longrightarrow} \underline{\mathcal{S}}\left(\underline{C^{0}}, \underline{C}\right) \cong \underline{C}$ equals the map $\imath_{0}$.

Q.E.D.

Corollary 3.20. Let $C$ be a df-abstract $\mathcal{S}$-clone. For all $n \in \mathbb{N}$, the map $\imath_{n}: C_{n} \longrightarrow \underline{C}$ is a monomorphism.

Theorem 3.21 (Embedding theorem). Let $\mathcal{S}$ be a cartesian closed category with colimits of $\omega$-chains of sections. For every df-abstract $\mathcal{S}$-clone $C$, the family $\rho$ provides an embedding of $C$ into the concrete discrete finitary $\mathcal{S}$-clone of operations $\underline{\mathcal{S}}\langle\underline{C}\rangle$ on $\underline{C}$ in $\underline{\mathcal{S}}$.

\section{Representation theorem}

We turn attention to the representation problem. Building on the embedding theorem of the previous section, for a given df-abstract $\mathcal{S}$-clone $C$, we seek a concrete $\mathcal{S}$-category with finite powers $\left(\underline{\mathscr{A}}_{C}, U_{C}: \underline{\mathscr{A}}_{C} \rightarrow \underline{\mathcal{S}}\right)$ and an object $A_{C} \in \underline{\mathscr{A}}_{C}$ together with an isomorphism $U_{C}\left(A_{C}\right) \cong \underline{C}$ so that the $\mathcal{S}$-clone embedding

$$
C \succ \mathcal{S}\langle\underline{C}\rangle
$$

factors through the sub $\mathcal{S}$-clone

$$
\underline{\mathscr{A}}_{C}\left\langle A_{C}\right\rangle \succ \mathcal{S}\langle\underline{C}\rangle
$$

yielding an $\mathcal{S}$-clone representation

$$
C \stackrel{\cong}{\longrightarrow} \mathscr{A}_{C}\left\langle A_{C}\right\rangle
$$

Notation 4.1. For an object $X$ in an enriched category $\underline{\mathscr{E}}$, we let $\operatorname{Endo}_{\underline{\mathscr{E}}}(X)$ denote the monoid of endomaps $\underline{\mathscr{E}}(X, X)$ on $X$.

The specific construction that we will provide is somewhat analogous to the construction of the Cayley representation of a monoid $M$, say with multiplication $m: M \otimes M \rightarrow M$, in a monoidal 
closed category with equalizers $\mathscr{E}$ into the monoid $\operatorname{Endo}_{\underline{\mathscr{E}}}(M)$

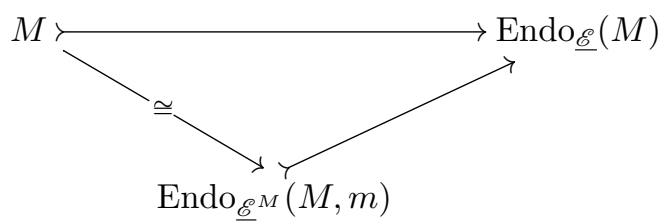

and the characterization of its image as the submonoid $\operatorname{Endo}_{\mathscr{E}} M(M, m)$, for $\underline{\mathscr{E}}^{M}$ the $\mathscr{E}$-category of (right) $M$-actions, of the equivariant endomaps on $M$ acting on itself with $m$. Indeed, for a df-abstract $\mathcal{S}$-clone $C$ in an enriching category $\mathcal{S}$ with appropriate structure to be presented shortly, we shall construct a monoid $\mathrm{M}(C)$ in $\mathcal{S}$ together with a (right) $\mathrm{M}(C)$-action $\mathrm{A}(C)$ on $\underline{C}$ and consider the concrete $\mathcal{S}$-category with finite powers $\underline{\mathcal{S}}^{\mathrm{M}(C)}$ of $\mathrm{M}(C)$-actions, establishing the following situation

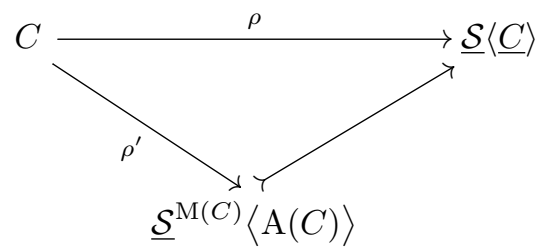

for $\rho^{\prime}$ a positive $\mathcal{S}$-clone representation, in the sense of having all its positive components invertible.

Notation 4.2. The internal hom of a monoidal left-closed category from an object $X$ to another one $Y$ will be denoted $[X, Y]$, and we write $\varepsilon_{X, Y}: X \otimes[X, Y] \rightarrow Y$ for its evaluation map.

The monoid $\mathrm{M}(C)$ will be a submonoid of the endo-hom monoid $[\underline{C}, \underline{C}]$ and its carrier

$$
\underline{C}^{\infty} \nmid[\underline{C}, \underline{C}]
$$

is to be thought of as consisting of assignments for variables. This will provide us with an action

$$
\underline{C} \otimes \underline{C}^{\infty} \rightarrow \underline{C}
$$

that, intuitively, substitutes in an operator according to a variable assignment. Variable assignments for operators of arbitrary arity need be of arbitrary length. To internalize such infinitary data in the enriching category we will be further requiring the following categorical structure.

Definition 4.3. A category $\mathscr{C}$ is said to have $\mathbb{N}$-powers whenever the functor

$$
\operatorname{Set}(\mathbb{N}, \mathscr{C}(-, X)): \mathscr{C}^{\mathrm{op}} \rightarrow \mathcal{S e t}
$$

is representable for all $X \in \mathscr{C}$. 
In a category with $\mathbb{N}$-powers, we write $\left\{\pi_{i}^{\infty}: X^{\infty} \rightarrow X\right\}_{i \in \mathbb{N}}$ for a family representing (9). If the category has finite powers, we note the fact to be used later that the $\omega^{\mathrm{op}}$-chain of projections

$$
\left\{X^{(k \rightarrow k+1)}=\left\langle\pi_{i}^{k+1}\right\rangle_{i \in[k]}: X^{k+1} \rightarrow X^{k}\right\}_{k \in \mathbb{N}}
$$

has as limit the cone $\kappa=\left\{\kappa_{k}=\left\langle\pi_{i}^{\infty}\right\rangle_{i \in[k]}: X^{\infty} \rightarrow X^{k}\right\}_{k \in \mathbb{N}}$.

Assumption 4.4. Henceforth, we work with an enriching monoidal biclosed category $\mathcal{S}$ assumed to have colimits of $\omega$-chains of sections and finite powers that preserve them, and also assumed to have $\mathbb{N}$-powers.

The next series of definitions construct the data (7) and (8). Proposition 4.8 and Corollary 4.13 show that they have the required structure.

Definition 4.5. For a df-abstract $\mathcal{S}$-clone $C$, let

$$
\lambda=\left\{\lambda_{n}: \underline{C}^{n} \rightarrow\left[C_{n}, \underline{C}\right]\right\}_{n \in \mathbb{N}}
$$

be given by left transposing each component of $\alpha$ in Definition 3.9.

By Proposition 3.10, the family $\lambda$ is a natural transformation $\underline{C}^{(-)} \stackrel{\dot{\rightarrow}}{\rightarrow}\left[C_{(-)}, \underline{C]}: \mathbb{F}^{\text {op }} \rightarrow \mathcal{S}\right.$ allowing us to proceed as follows.

Definition 4.6. For a df-abstract $\mathcal{S}$-clone $C$, we define

$$
\vec{\lambda}: \underline{C}^{\infty} \rightarrow[\underline{C}, \underline{C}]
$$

as the composite

$$
\underline{C}^{\infty}=\lim _{n \in \omega^{\mathrm{op}}} \underline{C}^{n} \stackrel{\lim _{n \in \omega^{\mathrm{op}} \lambda_{n}}}{\longrightarrow} \lim _{n \in \omega^{\mathrm{op}}}\left[C_{n}, \underline{C}\right]=\left[\operatorname{colim}_{n \in \omega} C_{n}, \underline{C}\right]=[\underline{C}, \underline{C}]
$$

and let

$$
\vec{\alpha}: \underline{C} \otimes \underline{C}^{\infty} \rightarrow \underline{C}
$$

be the left transpose of $\vec{\lambda}$.

Hence, we have the following situation:

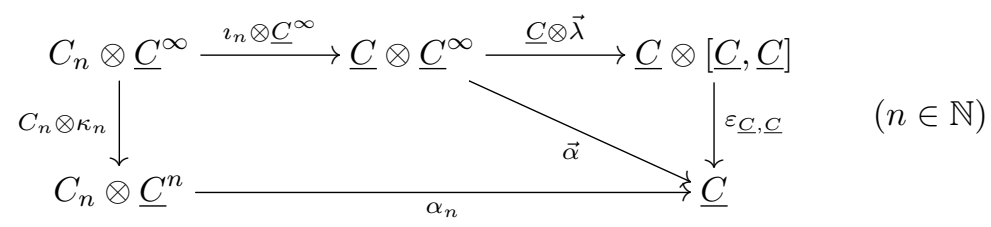

Definition 4.7. For a df-abstract $\mathcal{S}$-clone $C$, let

$$
\eta: I \rightarrow \underline{C}^{\infty}
$$


be the internalisation of the family

$$
\left\{\eta_{n}: I \longrightarrow \underline{C}^{n}\right\}_{n \in \mathbb{N}}
$$

introduced in Definition 3.13 and let

$$
\mu: \underline{C}^{\infty} \otimes \underline{C}^{\infty} \rightarrow \underline{C}^{\infty}
$$

be given by the composite

$$
\underline{C}^{\infty} \otimes \underline{C}^{\infty} \stackrel{\left\langle\pi_{i}^{\infty} \otimes \underline{C}^{\infty}\right\rangle_{i \in \mathbb{N}}}{\longrightarrow}\left(\underline{C} \otimes \underline{C}^{\infty}\right)^{\infty} \stackrel{\vec{\alpha}^{\infty}}{\longrightarrow} \underline{C}^{\infty} .
$$

Proposition 4.8. For a df-abstract $\mathcal{S}$-clone $C$, the structure $\mathrm{M}(C)=\left(\underline{C}^{\infty}, \eta, \mu\right)$ is a monoid in $\mathcal{S}$ and the structure $\mathrm{A}(C)=(\underline{C}, \vec{\alpha})$ is an $\mathrm{M}(C)$-action.

Proof. Follows from Proposition B.1 in Appendix B.

Q.E.D.

Remark 4.9. We note that our construction is related to the one introduced by Sangalli [17] for the purpose of clone representation in the category of sets. The main point of difference between the two approaches is in the construction of the monoid on which to take actions. Whereas Sangalli takes it to be freely generated by some basic elements within a certain monoid of endomaps, I have explicitly constructed a different one. Specifically, for an abstract clone $C$, the monoid $M_{C}$ of Sangalli embeds in our monoid $\mathrm{M}(C)$ as the submonoid consisting of the sequences $\left\langle c_{i}\right\rangle_{i \in \mathbb{N}} \in \underline{C}^{\infty}$ such that the set of indices $\left\{i \in \mathbb{N} \mid c_{i} \neq \imath_{i+1}\left(\nu_{i}^{i+1}\right)\right\}$ is finite.

Our construction is functorial.

Definition 4.10. For a monoidal category $(\mathcal{S}, I, \otimes)$, we let $\mathcal{A} \boldsymbol{c t}(\mathcal{S})$ be the category of $\mathcal{S}$-actions and their homomorphisms, with objects $(\mathrm{M},(A, \alpha))$ given by a monoid $\mathrm{M}=(M, e: I \rightarrow M, m$ : $M \otimes M \rightarrow M)$ together with an M-action $(A, \alpha: A \otimes M \rightarrow A)$ in $\mathcal{S}$ and with morphisms $(h, f):$ $(\mathrm{M},(A, \alpha)) \rightarrow\left(\mathrm{M}^{\prime},\left(A^{\prime}, \alpha^{\prime}\right)\right)$ consisting of a monoid homomorphism $h: \mathrm{M} \rightarrow \mathrm{M}^{\prime}$ and an $\mathrm{M}$-action homomorphism $f:(A, \alpha) \rightarrow\left(A^{\prime}, \alpha^{\prime} \circ\left(A^{\prime} \otimes h\right)\right)$.

Proposition 4.11. The mapping $C \longmapsto(\mathrm{M}(C), \mathrm{A}(C))$ extends to a functor $\boldsymbol{A} \boldsymbol{b} \mathcal{C}(\mathcal{S}) \rightarrow \boldsymbol{A c t}(\mathcal{S})$ from the category of df-abstract $\mathcal{S}$-clones to the category of $\mathcal{S}$-actions.

Proposition 4.12. For a df-abstract $\mathcal{S}$-clone $C$, the map $\vec{\lambda}: \underline{C}^{\infty} \rightarrow[\underline{C}, \underline{C}]$ in Definition 4.6 is a section.

Proof. See Appendix B.

Q.E.D.

Corollary 4.13. For a df-abstract $\mathcal{S}$-clone $C$, the map $\vec{\lambda}$ is a monoid homomorphism from $\mathrm{M}(C)$ to the endo-hom monoid $[\underline{C}, \underline{C}]$. Thus, $\mathrm{M}(C)$ is a submonoid of $[\underline{C}, \underline{C}]$.

Let us now recall some background on actions in the enriched context. For a monoid $M$ in a monoidal (right) closed category with equalizers $\mathcal{S}$, the $\mathcal{S}$-category $\underline{\mathcal{S}}^{M}$ has objects given by (right) $M$-actions $(A, a: A \otimes M \rightarrow A)$ and hom-objects given by the equalizer

$$
\underline{\mathcal{S}}^{M}((X, x),(Y, y)) \succ \underline{\mathcal{S}}(X, Y) \longrightarrow \mathcal{S}(X \otimes M, Y)
$$


where the parallel pair consists of the right transposes of

$$
\underline{\mathcal{S}}(X, Y) \otimes X \otimes M \stackrel{\varepsilon_{X, Y} \otimes M}{\longrightarrow} Y \otimes M \stackrel{y}{\longrightarrow} Y
$$

and

$$
\underline{\mathcal{S}}(X, Y) \otimes X \otimes M \stackrel{\underline{\mathcal{S}}(X, Y) \otimes x}{\longrightarrow} \underline{\mathcal{S}}(X, Y) \otimes X \stackrel{\varepsilon_{X, Y}}{\longrightarrow} Y .
$$

Identities and composition in $\underline{\mathcal{S}}^{M}$ are restrictions of those of $\underline{\mathcal{S}}$. Furthemore, if $\mathcal{S}$ has finite powers, then so does the $\mathcal{S}$-category $\underline{\mathcal{S}}^{M}$; and, for $n \in \mathbb{N}$, these are given by

$$
(X, x)^{n}=\left(X^{n}, X^{n} \otimes M \stackrel{\left\langle\pi_{i}^{n} \otimes M\right\rangle_{i \in[n]}}{\longrightarrow}(X \otimes M)^{n} \stackrel{x^{n}}{\longrightarrow} X^{n}\right) .
$$

For an $M$-action $A=(X, x)$ in $\mathcal{S}$, the family of equalizers

$$
e=\left\{e_{n}: \underline{\mathcal{S}}^{M}\left(A^{n}, A\right) \succ \underline{\mathcal{S}}\left(X^{n}, X\right)\right\}_{n \in \mathbb{N}}
$$

is an embedding $\underline{\mathcal{S}}^{M}\langle A\rangle \succ \underline{\mathcal{S}}\langle X\rangle$ of concrete discrete finitary $\mathcal{S}$-clones of operations.

Assumption 4.14. Henceforth, we work with an enriching monoidal biclosed category $\mathcal{S}$ assumed to have colimits of $\omega$-chains of sections and finite powers that preserve them, and also assumed to have $\mathbb{N}$-powers and equalizers.

We establish (6).

Proposition 4.15. Let $C$ be an $\mathcal{S}$-clone. For $n \in \mathbb{N}$, the morphism $\rho_{n}: C_{n} \rightarrow \underline{\mathcal{S}}\left(\underline{C}^{n}, \underline{C}\right)$ restricts, along the monomorphism $e_{n}: \underline{\mathcal{S}}^{\mathrm{M}(C)}\left(\mathrm{A}(C)^{n}, \mathrm{~A}(C)\right) \succ \underline{\mathcal{S}}\left(\underline{C}^{n}, \underline{C}\right)$, to a morphism

$$
\rho_{n}^{\prime}: C_{n} \rightarrow \underline{\mathcal{S}}^{\mathrm{M}(C)}\left(\mathrm{A}(C)^{n}, \mathrm{~A}(C)\right) .
$$

Proof. See Appendix C.

Q.E.D.

Note that, by Proposition 3.15 , the morphism $\rho_{n}^{\prime}$, for all $1 \leq n \in \mathbb{N}$, is a section with retraction

$$
\underline{\mathcal{S}}^{\mathrm{M}(C)}\left(\mathrm{A}(C)^{n}, \mathrm{~A}(C)\right) \succ \stackrel{e_{n}}{\longrightarrow} \underline{\mathcal{S}}\left(\underline{C}^{n}, \underline{C}\right) \stackrel{\underline{\mathcal{S}}\left(\eta_{n}, \jmath_{n}\right)}{\longrightarrow} \underline{\mathcal{S}}\left(I, C_{n}\right) \cong C_{n} .
$$

The family (13) provides a positive $\mathcal{S}$-clone embedding

$$
\rho^{\prime}: C \longrightarrow \underline{\mathcal{S}}^{\mathrm{M}(C)}\langle\mathrm{A}(C)\rangle
$$

that yields our representation theorem. Its proof is technical and deferred to Appendix C.

Definition 4.16. A (positive) representation of df-abstract enriched clones is an homomorphism for which every (positive) component is an isomorphism. 
Theorem 4.17 (Representation theorem). Let $\mathcal{S}$ be a monoidal biclosed category with colimits of $\omega$-chains of sections and finite powers that preserve them, and with $\mathbb{N}$-powers and equalizers. For every df-abstract $\mathcal{S}$-clone $C$ the family $\rho^{\prime}$ is a positive representation of $C$ as the concrete discrete finitary $\mathcal{S}$-clone of operations $\underline{\mathcal{S}}^{\mathrm{M}(C)}\langle\mathrm{A}(C)\rangle$ on the $\mathrm{M}(C)$-action $\mathrm{A}(C)$ in $\underline{\mathcal{S}}^{\mathrm{M}(C)}$.

Remark 4.18. Note that the positive representation $\rho^{\prime}$ is a representation iff the commuting diagram

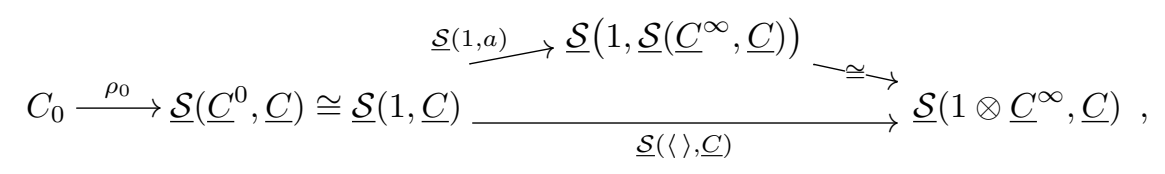

where $a: \underline{C} \rightarrow \underline{\mathcal{S}}\left(\underline{C}^{\infty}, \underline{C}\right)$ denotes the right transpose of $\vec{\alpha}: \underline{C} \otimes \underline{C}^{\infty} \rightarrow \underline{C}$, is an equalizer.

Corollary 4.19. For every object $X$ of an $\mathcal{S}$-category $\underline{\mathscr{E}}$ with finite powers,

$$
\operatorname{Endo}_{\underline{\mathscr{E}}}\left(X^{n}\right) \cong \operatorname{Endo}_{\underline{\mathcal{S}}^{\mathrm{M}(\underline{\mathscr{E}}\langle X\rangle)}}\left(\mathrm{A}(\underline{\mathscr{E}}\langle X\rangle)^{n}\right)
$$

for all $n \in \mathbb{N}$.

\section{$5 \quad$ Enriched abstract clones with discrete countable arities}

We conclude the paper outlining a conceptual framework for the constructions and associated calculations involved in the embedding and representation theorems. This requires extending the notion of discrete finitary enriched abstract clone to also include operators of infinite countable arity.

Assumption 5.1. Henceforth, we work with an enriching monoidal category $\mathcal{S}$ with countable powers.

Notation 5.2. We let $\overline{\mathbb{N}}=\mathbb{N} \cup\{\infty\}$, with $n<\infty$ for all $n \in \mathbb{N}$, and set $[\infty]=\mathbb{N}$.

Definition 5.3 (Discrete countable enriched abstract clones). Let $\mathcal{S}$ be a monoidal category with countable powers.

A dc-abstract $\mathcal{S}$-clone $(D, \nu, \varsigma)$ is a structure

$$
\begin{gathered}
D=\left\{D_{n} \in \mathcal{S}\right\}_{n \in \overline{\mathbb{N}}}, \nu=\left\{\left\langle\nu_{i}^{n}\right\rangle_{i \in[n]}: I \longrightarrow D_{n}{ }^{n} \text { in } \mathcal{S}\right\}_{n \in \overline{\mathbb{N}}}, \\
\varsigma=\left\{\varsigma_{m, n}: D_{m} \otimes D_{n}{ }^{m} \longrightarrow D_{n} \text { in } \mathcal{S}\right\}_{m, n \in \overline{\mathbb{N}}}
\end{gathered}
$$

satisfying the laws of Definition 2.3.

An homomorphism $h:(D, \nu, \varsigma) \longrightarrow\left(D^{\prime}, \nu^{\prime}, \varsigma^{\prime}\right)$ of dc-abstract $\mathcal{S}$-clones is a family

$$
h_{n}: D_{n} \rightarrow D_{n}^{\prime} \text { in } \mathcal{S}(n \in \overline{\mathbb{N}})
$$


satisfying the laws of Definition 2.10.

We let $\mathcal{A} b \mathcal{C}_{\infty}(S)$ be the category of dc-abstract $\mathcal{S}$-clones and their homomorphisms.

Remark 5.4. The notion of dc-abstract enriched clone above is essentially a rewording of Power's notion of discrete countable enriched Lawvere theory (see [15, Definition 4]) in the language of abstract clones.

For a dc-abstract $\mathcal{S}$-clone $(D, \nu, \varsigma)$, the structure

$$
\left(D_{\infty},\left\langle\nu_{i}^{\infty}\right\rangle_{i \in \mathbb{N}}: I \rightarrow D_{\infty}^{\infty}, \varsigma_{\infty, \infty}: D_{\infty} \otimes D_{\infty}^{\infty} \rightarrow D_{\infty}\right)
$$

is a unitary Menger algebra of infinite countable rank and thus has an associated monoid action as follows.

Proposition 5.5. For a dc-abstract $\mathcal{S}$-clone $(D, \nu, \varsigma)$, the structure

$$
\mathrm{M}_{\infty}(D)=\left(D_{\infty}^{\infty}, \eta: I \rightarrow D_{\infty}^{\infty}, \mu: D_{\infty}^{\infty} \otimes D_{\infty}^{\infty} \rightarrow D_{\infty}^{\infty}\right)
$$

where

$$
\eta=\left\langle\nu_{i}^{\infty}\right\rangle_{i \in \mathbb{N}}, \quad \mu=\left(D_{\infty}^{\infty} \otimes D_{\infty}^{\infty} \stackrel{\left\langle\pi_{i}^{\infty} \otimes D_{\infty}^{\infty}\right\rangle_{i \in \mathbb{N}}}{\longrightarrow}\left(D_{\infty} \otimes D_{\infty}^{\infty}\right)^{\infty} \stackrel{\varsigma_{\infty, \infty}^{\infty}}{\longrightarrow} D_{\infty}\right)
$$

is a monoid and the structure

$$
\mathrm{A}_{\infty}(D)=\left(D_{\infty}, \varsigma_{\infty, \infty}: D_{\infty} \otimes D_{\infty}^{\infty} \rightarrow D_{\infty}\right)
$$

an $\mathrm{M}_{\infty}(D)$-action.

Proposition 5.6. The mapping $D \mapsto\left(\mathrm{M}_{\infty}(D), \mathrm{A}_{\infty}(D)\right)$ extends to a functor $\mathcal{A} b \mathcal{C}_{\infty}(\mathcal{S}) \rightarrow$ $\mathcal{A} \boldsymbol{c t}(\mathcal{S})$ from the category of dc-abstract $\mathcal{S}$-clones to the category of $\mathcal{S}$-actions.

Assumption 5.7. Henceforth, we work with an enriching monoidal closed category $\mathcal{S}$ with countable powers.

For a dc-abstract $\mathcal{S}$-clone $(D, \nu, \varsigma)$, we have a discrete countable $\mathcal{S}$-clone of operations $\mathcal{S}\left\langle D_{\infty}\right\rangle$ with carrier $\left\{\underline{\mathcal{S}}\left(D_{\infty}{ }^{n}, D_{\infty}\right)\right\}_{n \in \overline{\mathbb{N}}}$, and the right transposes

$$
\rho_{n}: D_{n} \rightarrow \underline{\mathcal{S}}\left(D_{\infty}^{n}, D_{\infty}\right) \quad(n \in \overline{\mathbb{N}})
$$

of the substitution maps

$$
\varsigma_{n, \infty}: D_{n} \otimes D_{\infty}^{n} \rightarrow D_{\infty} \quad(n \in \overline{\mathbb{N}})
$$

are the components of an homomorphism of dc-abstract $\underline{\mathcal{S}}$-clones

$$
\rho: D \rightarrow \underline{\mathcal{S}}\left\langle D_{\infty}\right\rangle .
$$

Every positive component of $\rho$ is a section and an embedding theorem follows. 
Theorem 5.8 (Embedding theorem). Let $\mathcal{S}$ be a monoidal closed category with countable powers. For every dc-abstract $\mathcal{S}$-clone $D$, the family $\rho$ provides a positive embedding of $D$ into the concrete discrete countable $\mathcal{S}$-clone of operations $\underline{\mathcal{S}}\left\langle D_{\infty}\right\rangle$ on $D_{\infty}$ in $\underline{\mathcal{S}}$. Moreover, this is an embedding whenever $D_{0}$ is inhabited.

Analogously to the development of the previous section, the embedding may be refined to provide a representation.

Assumption 5.9. Henceforth, we work with an enriching monoidal closed category $\mathcal{S}$ with countable powers and equalizers.

For a dc-abstract $\mathcal{S}$-clone $D$, we have a factorization as follows

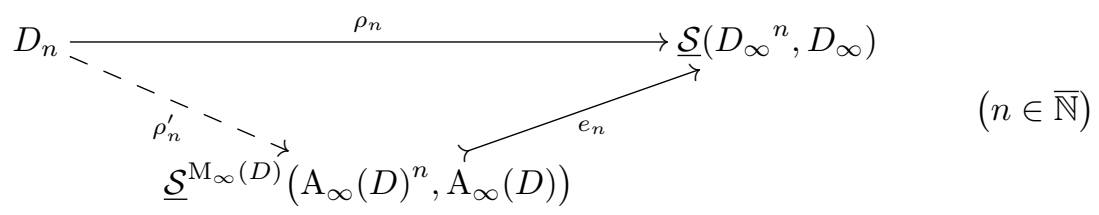

that yields a positive Cayley-style representation.

Theorem 5.10 (Representation theorem). Let $\mathcal{S}$ be a monoidal closed category with countable powers and equalizers. For every dc-abstract $\mathcal{S}$-clone $D$ the family $\rho^{\prime}$ is a positive representation of $D$ as the concrete discrete countable $\mathcal{S}$-clone of operations $\underline{\mathcal{S}}^{\mathrm{M}_{\infty}(D)}\left\langle\mathrm{A}_{\infty}(D)\right\rangle$ on the $\mathrm{M}_{\infty}(D)$ action $\mathrm{A}_{\infty}(D)$ in $\underline{\mathcal{S}}^{\mathrm{M}_{\infty}(D)}$.

Finally, we show how the representation theorem for df-abstract enriched clones may be recovered from that for dc-abstract ones

Assumption 5.11. Henceforth, we work with an enriching monoidal biclosed category $\mathcal{S}$ with countable powers and colimits of $\omega$-chains of sections that are preserved by finite powers.

Every df-abstract $\mathcal{S}$-clone $C$ can be extended to a dc-abstract one, which we shall denote $C^{\#}$, with object of operators of infinite arity given by $\underline{C}$ (Definition 3.5), and associated variables

$$
\left\langle\underline{\nu}_{i}\right\rangle_{i \in \mathbb{N}}: I \rightarrow \underline{C}^{\infty} \quad \text { (Definition 3.13) }
$$

and substitution maps, for $n \in \mathbb{N}$, as follows

$$
\begin{array}{ll}
\alpha_{n}: C_{n} \otimes \underline{C}^{n} \rightarrow \underline{C} & \text { (Definition 3.9) }, \\
\vec{\alpha}: \underline{C} \otimes \underline{C}^{\infty} \rightarrow \underline{C} & \text { (Definition 4.6) }, \\
\varsigma_{\infty, n}: \underline{C} \otimes C_{n}{ }^{\infty} \rightarrow C_{n} &
\end{array}
$$


where the last map is the unique such that the diagram

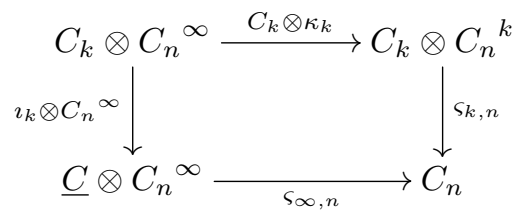

commutes for all $k \in \mathbb{N}$. This construction is universal.

Proposition 5.12. The forgetful functor $\mathcal{A b C}_{\infty}(\mathcal{S}) \rightarrow \mathcal{A} b \mathcal{C}(\mathcal{S})$ has a left adjoint, with the free dc-abstract $\mathcal{S}$-clone on a df-abstract $\mathcal{S}$-clone $C$ given by $C^{\#}$.

For a df-abstract $\mathcal{S}$-clone $C$, we have that

$$
\mathrm{M}_{\infty}\left(C^{\#}\right)=\mathrm{M}(C), \quad \mathrm{A}_{\infty}\left(C^{\#}\right)=\mathrm{A}(C)
$$

and that the representation of $C^{\#}$ provides the representation of $C$.

\section{Appendix}

\section{A Homomorphism property of $\rho$}

Assume the context of Section 3.

Proposition A.1. Let $C$ be a df-abstract $\mathcal{S}$-clone with variables $\nu$. For $n \in \mathbb{N}$ and $i \in[n]$, the diagram

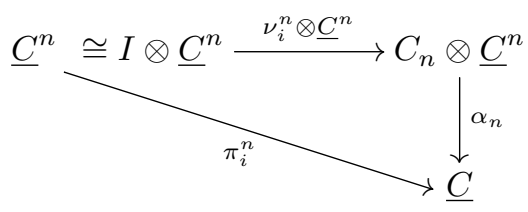

commutes.

Proof. Because the diagram

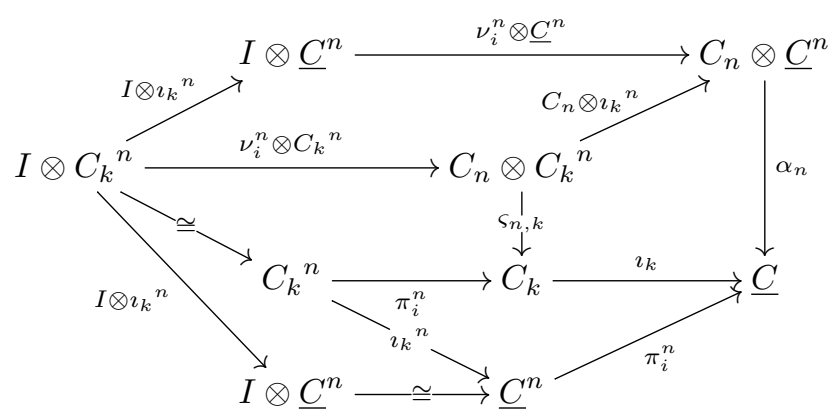

is commutative for all $k \in \mathbb{N}$.

Q.E.D. 
Lemma A.2. Let $C$ be a df-abstract $\mathcal{S}$-clone. For $m, n \in \mathbb{N}$, the diagram

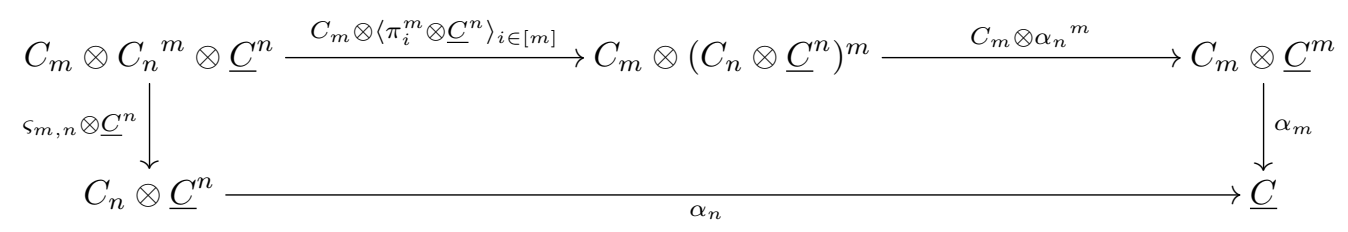

commutes.

Proof. Because the diagrams

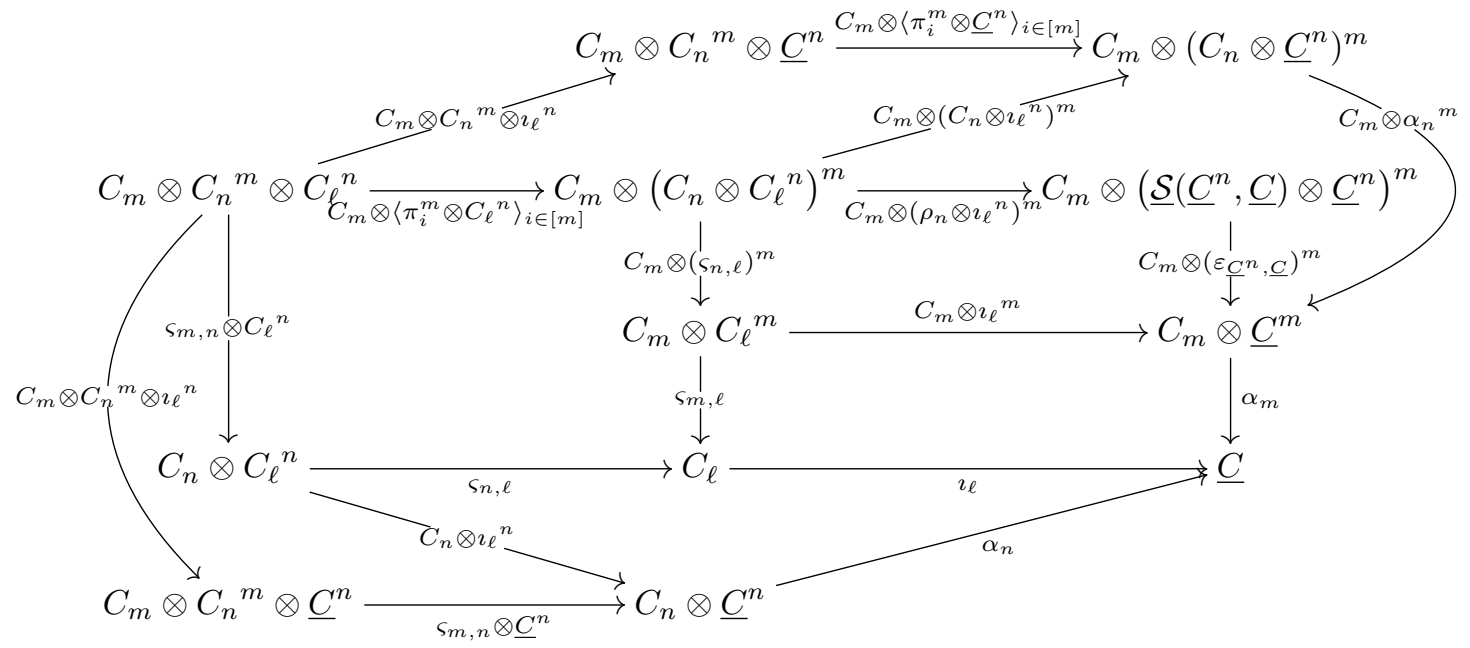

are commutative for all $\ell \in \mathbb{N}$.

Q.E.D.

Proof of Proposition 3.12. The variables $\varpi_{i}^{n}: I \rightarrow \underline{\mathcal{S}}\left(\underline{C}^{n}, \underline{C}\right)(n \in \mathbb{N}, i \in[n])$ are given by the right transpose of the composite $I \otimes \underline{C}^{n} \cong \underline{C}^{n} \stackrel{\pi_{i}^{n}}{\longrightarrow} \underline{C}$, and the diagram

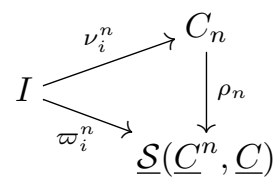

commutes by Proposition A.1.

For $m, n \in \mathbb{N}$, the diagram

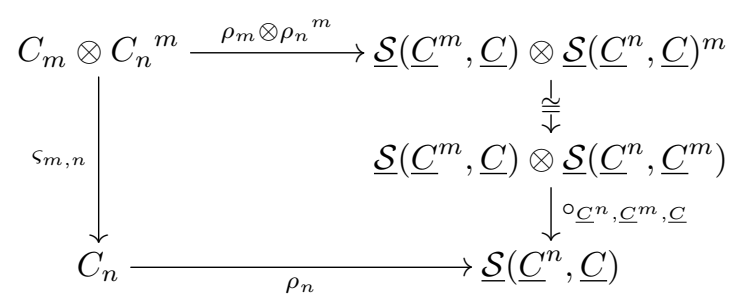


commutes iff so does

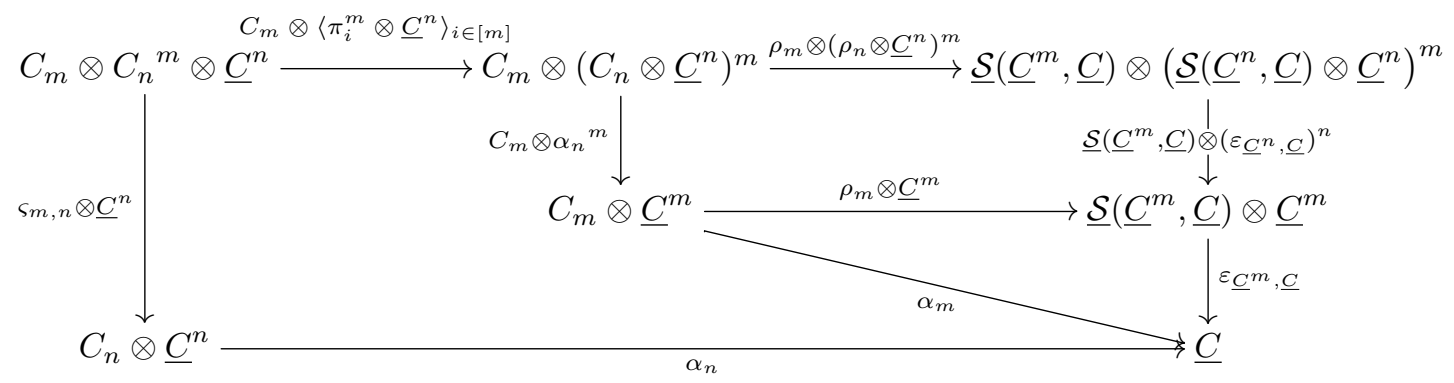

and this is the case by Lemma A.2.

Q.E.D.

\section{B Monoid and action structures of $\mathrm{M}(C)$ and $\mathrm{A}(C)$}

Assume the contexts of Sections 3 and 4 .

Proposition B.1. For a df-abstract $\mathcal{S}$-clone $C$, the following diagrams commute.
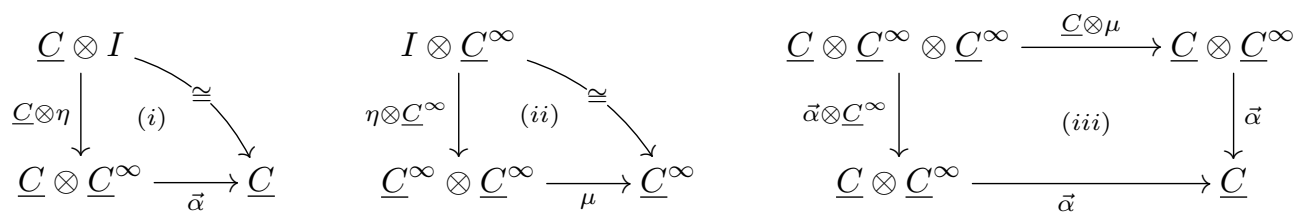

Proof. Diagram (i) commutes because, by Proposition 3.14 and Diagram (10), so do

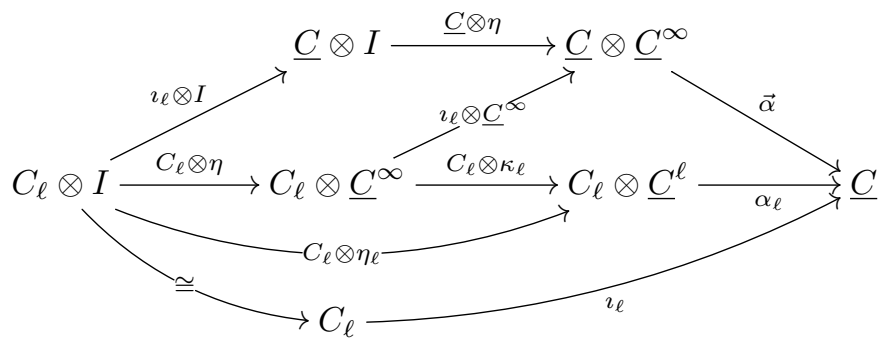

for all $\ell \in \mathbb{N}$. 
Diagram (ii) commutes because, by Definition 3.13, Proposition A.1 and Diagram (10), so do

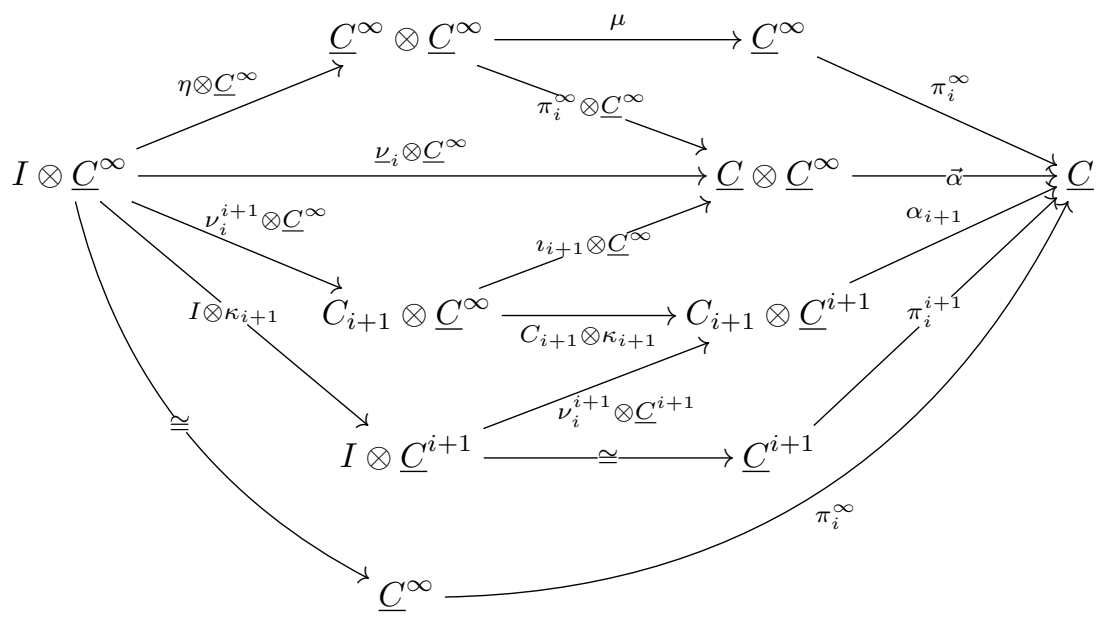

for all $i \in \mathbb{N}$.

We show that Diagram (iii) commutes in a series of steps. First, since by Diagram (10), we have the commutativity of

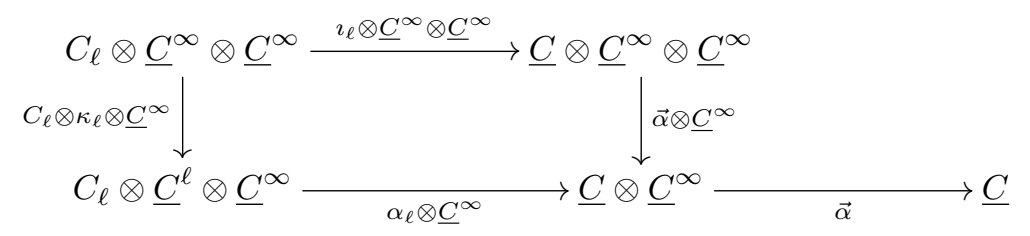

and

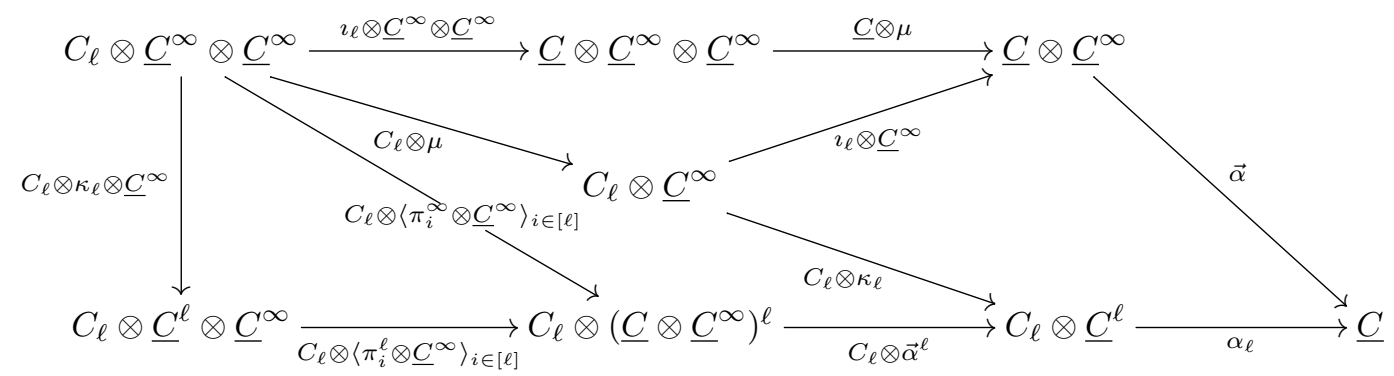

for all $\ell \in \mathbb{N}$, it is enough to show that the lower horizontal composites of (14) and (15) above are 
equal. Again, since by Diagram (10), we have that

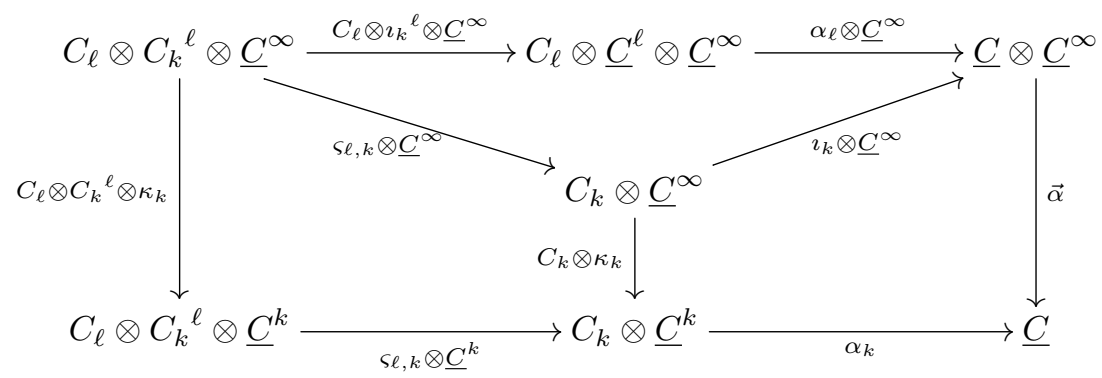

and that

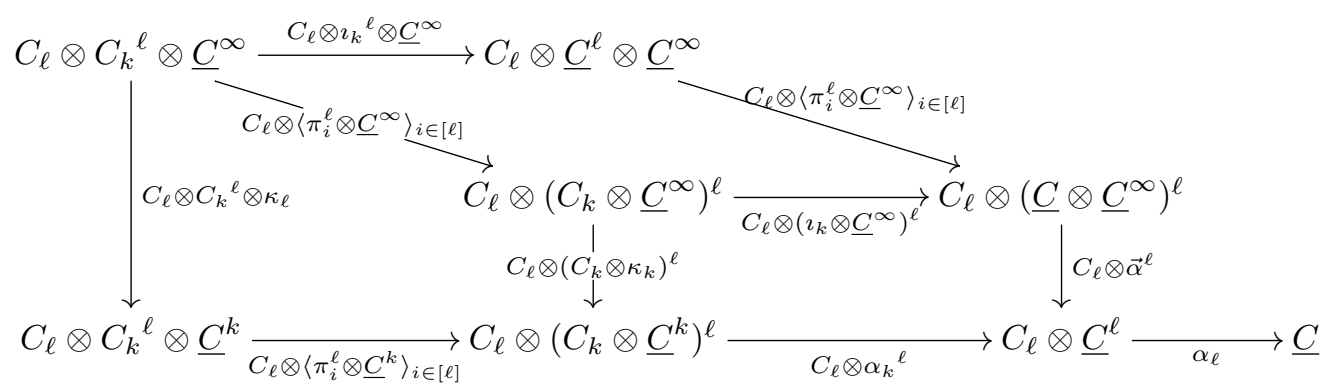

for all $k \in \mathbb{N}$, it is enough to show that the lower horizontal composites of (16) and (17) are equal. This is the case by Lemma A.2.

Proof of Proposition 4.12. The composite

$$
[\underline{C}, \underline{C}] \cong I \otimes[\underline{C}, \underline{C}] \stackrel{\left\langle\underline{\nu}_{i} \otimes[\underline{C}, \underline{C}]\right\rangle_{i \in \mathbb{N}}}{\longrightarrow}(\underline{C} \otimes[\underline{C}, \underline{C}])^{\infty} \stackrel{\left(\varepsilon_{\underline{C}, \underline{C}}\right)^{\infty}}{\longrightarrow} \underline{C}^{\infty}
$$

provides a retraction for $\vec{\lambda}$. 
Indeed, for all $i \in \mathbb{N}$, we have the following commutative diagram

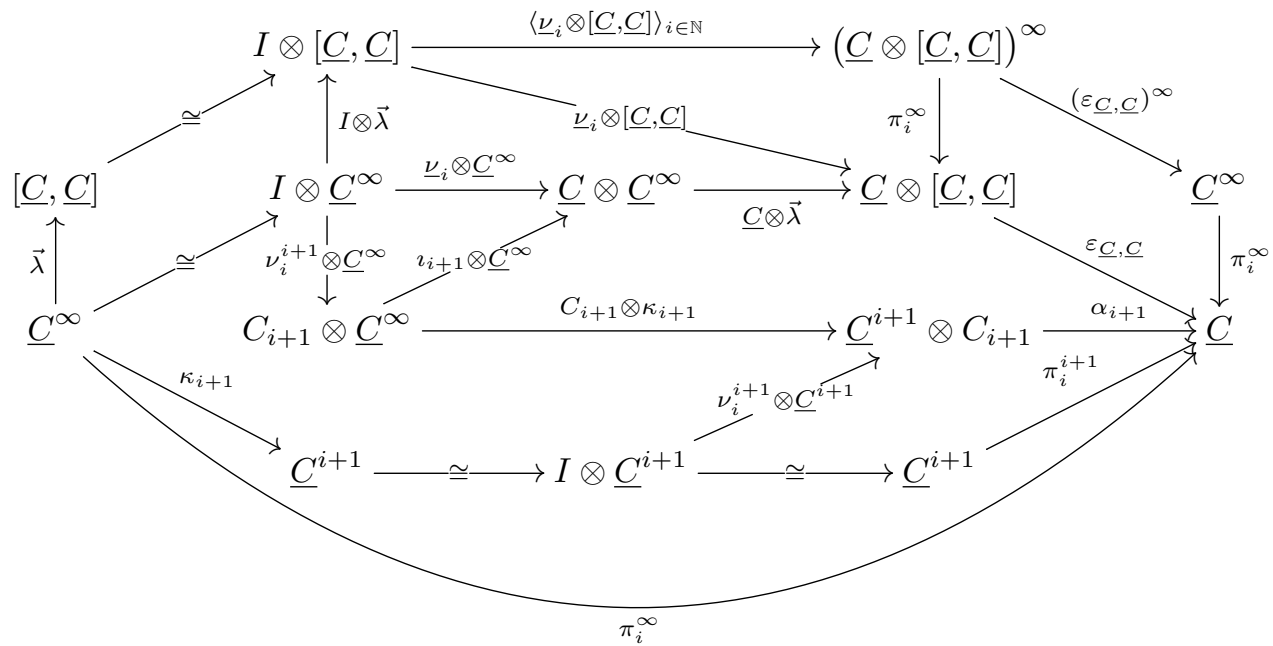

using Definition 3.13, Diagram (10), and Proposition A.1.

Q.E.D.

\section{Positive representation structure of $\rho^{\prime}$}

Assume the contexts of Sections 3 and 4 .

Proof of Proposition 4.15. Let $n \in \mathbb{N}$. We show that $\rho_{n}$ equalizes the parallel pair

$$
\underline{\mathcal{S}}\left(\underline{C}^{n}, \underline{C}\right) \rightrightarrows \underline{\mathcal{S}}\left(\underline{C}^{n} \otimes \underline{C}^{\infty}, \underline{C}\right)
$$

given by the right transposes of

$$
\underline{\mathcal{S}}\left(\underline{C}^{n}, \underline{C}\right) \otimes \underline{C}^{n} \otimes \underline{C}^{\infty} \stackrel{\varepsilon_{\underline{C}^{n}, \underline{C}} \otimes \underline{C}^{\infty}}{\longrightarrow} \underline{C} \otimes \underline{C}^{\infty} \stackrel{\vec{\alpha}}{\longrightarrow} \underline{C}
$$

and

$$
\underline{\mathcal{S}}\left(\underline{C}^{n}, \underline{C}\right) \otimes \underline{C}^{n} \otimes \underline{C}^{\underline{\mathcal{S}}\left(\underline{C}^{n}, \underline{C}\right) \otimes\left\langle\pi_{i}^{n} \otimes \underline{C}^{\infty}\right\rangle_{i \in[n]} \longrightarrow} \underline{\mathcal{S}}\left(\underline{C}^{n}, \underline{C}\right) \otimes\left(\underline{C} \otimes \underline{C}^{\infty}\right)^{n} \stackrel{\underline{\mathcal{S}}\left(\underline{C}^{n}, \underline{C}\right) \otimes \vec{\alpha}^{n}}{\longrightarrow} \underline{\mathcal{S}}\left(\underline{C}^{n}, \underline{C}\right) \otimes \underline{C}^{n} \stackrel{\varepsilon_{\underline{C}^{n}, \underline{C}}}{\longrightarrow} \underline{C} .
$$


To see this, note first that, for all $\ell \in \mathbb{N}$, we have that

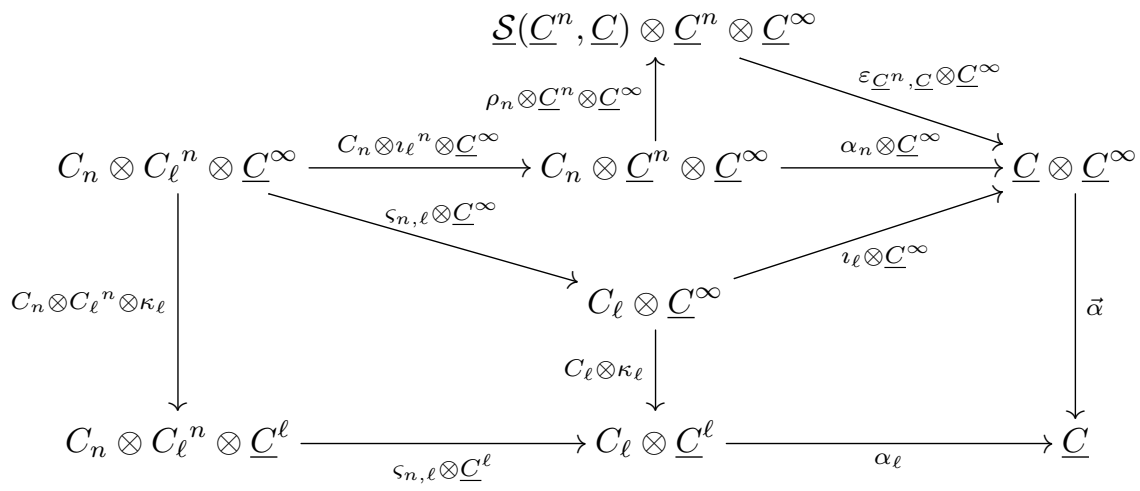

and that

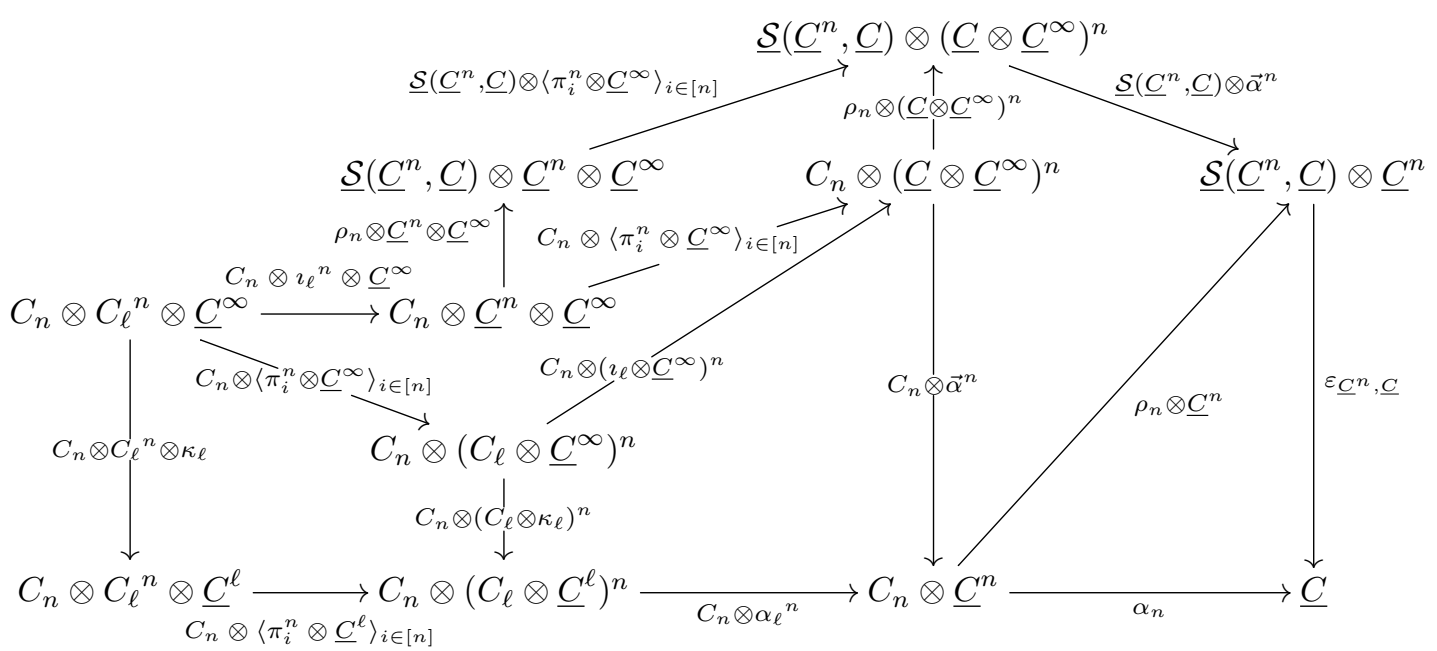

Then, since by Lemma A.2 the above two lower horizontal composites are equal, we are done. Q.E.D.

Proposition C.1. Let $C$ be a df-abstract $\mathcal{S}$-clone. For $i \in \mathbb{N}$,

$$
\pi_{i}^{\infty}=\left(\underline{C}^{\infty} \cong I \otimes \underline{C}^{\infty} \stackrel{\underline{\nu}_{i} \otimes \underline{C}^{\infty}}{\longrightarrow} \underline{C} \otimes \underline{C}^{\infty} \stackrel{\vec{\alpha}}{\longrightarrow} \underline{C}\right)
$$


Proof. Because the diagram

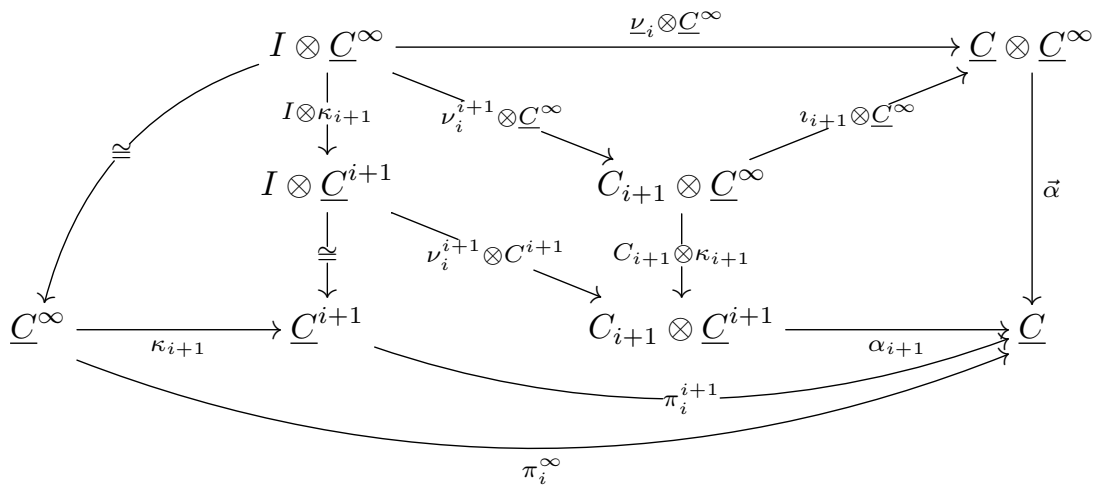

commutes for all $i \in \mathbb{N}$.

Q.E.D.

Definition C.2. Let $C$ be a df-abstract $\mathcal{S}$-clone. For $1 \leq n \in \mathbb{N}$, let $\gamma_{n}: \underline{C}^{n} \longrightarrow \underline{C}^{\infty}$ be the unique mediating map from the cone $\left\{\underline{C}^{(i \rightarrow n)}: \underline{C}^{n} \rightarrow \underline{C}^{i}\right\}_{i \in \mathbb{N}}$ of the $\omega^{\text {op }}$-chain $\left\{\underline{C}^{(i \rightarrow i+1)}: \underline{C}^{i+1} \rightarrow \underline{C}^{i}\right\}_{i \in \mathbb{N}}$ to the limiting cone $\left\{\kappa_{i}: \underline{C}^{\infty} \longrightarrow \underline{C}^{i}\right\}_{i \in \mathbb{N}}$.

Proposition C.3. Let $C$ be a df-abstract $\mathcal{S}$-clone. For $1 \leq n \in \mathbb{N}$, the diagram

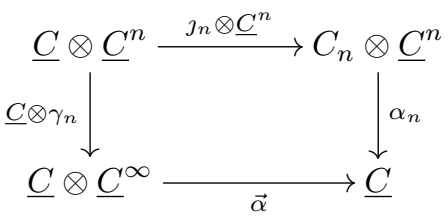

commutes.

Proof. Because, using Proposition 3.10, we have that

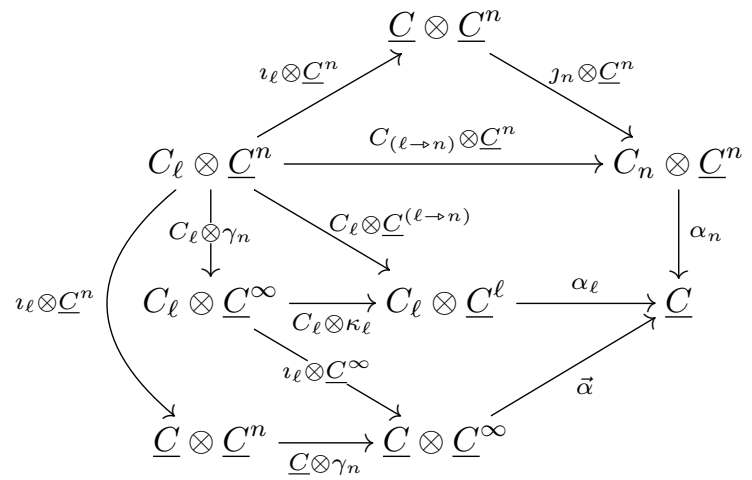

for all $\ell \in \mathbb{N}$. 
Proposition C.4. For a df-abstract $\mathcal{S}$-clone $C$, the embedding $\rho^{\prime}: C \succ \underline{\mathcal{S}}^{\mathrm{M}(C)}\langle\mathrm{A}(C)\rangle$ is a positive representation.

Proof. For $1 \leq n \in \mathbb{N}$, we show that, for $\tau_{n}$ the retraction $\underline{\mathcal{S}}\left(\underline{C}^{n}, \underline{C}\right) \stackrel{\underline{\mathcal{S}}\left(\eta_{n}, \jmath_{n}\right)}{\longrightarrow} \underline{\mathcal{S}}\left(I, C_{n}\right) \cong C_{n}$ of $\rho_{n}$ given in Proposition 3.15, we have $\rho_{n} \tau_{n} e_{n}=e_{n}: \underline{\mathcal{S}}^{\mathrm{M}(C)}\left(\overline{\mathrm{A}}(C)^{n}, \mathrm{~A}(C)\right) \succ \underline{\mathcal{S}}\left(\underline{C}^{n}, \underline{C}\right)$; so that, since $\rho_{n}=e_{n} \rho_{n}^{\prime}$, it follows that $\rho_{n}^{\prime} \tau_{n} e_{n}$ is the identity and hence that the section $\rho_{n}^{\prime}$ is also a retraction.

The calculation below shows that $\rho_{n} \tau_{n} e_{n}$ and $e_{n}$ have the same transpose. It relies on the definitions of $\rho_{n}$ (Definition 3.9), $e_{n}$ (see (11) and (12)), $\eta_{n}$ (Definition 3.13), $\gamma_{n}$ (Definition C.2), and on Propositions C.1 and C.3.

$$
\begin{aligned}
& \underline{\mathcal{S}}^{\mathrm{M}(C)}\left(\mathrm{A}(C)^{n}, \mathrm{~A}(C)\right) \otimes \underline{C}^{n} \stackrel{e_{n} \otimes \mathrm{id}}{\longrightarrow} \mathcal{S}\left(\underline{C}^{n}, \underline{C}\right) \otimes \underline{C}^{n} \cong \underline{\mathcal{S}}\left(\underline{C}^{n}, \underline{C}\right) \otimes I \otimes \underline{C}^{n} \\
& \stackrel{\mathrm{id} \otimes \eta_{n} \otimes \mathrm{id}}{\longrightarrow} \mathcal{S}\left(\underline{C}^{n}, \underline{C}\right) \otimes \underline{C}^{n} \otimes \underline{C}^{n} \stackrel{\varepsilon_{\underline{C}^{n}, \underline{C}} \otimes \mathrm{id}}{\longrightarrow} \underline{C} \otimes \underline{C}^{n} \stackrel{\jmath_{n} \otimes \mathrm{id}}{\longrightarrow} C_{n} \otimes \underline{C}^{n} \\
& \stackrel{\rho_{n} \otimes \mathrm{id}}{\longrightarrow} \underline{\mathcal{S}}\left(\underline{C}^{n}, \underline{C}\right) \otimes \underline{C}^{n} \stackrel{\varepsilon_{\underline{C}^{n}, \underline{C}}}{\longrightarrow} \underline{C} \\
& =\underline{\mathcal{S}}^{\mathrm{M}(C)}\left(\mathrm{A}(C)^{n}, \mathrm{~A}(C)\right) \otimes \underline{C}^{n} \stackrel{e_{n} \otimes \mathrm{id}}{\longrightarrow} \underline{\mathcal{S}}\left(\underline{C}^{n}, \underline{C}\right) \otimes \underline{C}^{n} \cong \underline{\mathcal{S}}\left(\underline{C}^{n}, \underline{C}\right) \otimes I \otimes \underline{C}^{n} \\
& \stackrel{\mathrm{id} \otimes \eta_{n} \otimes \mathrm{id}}{\longrightarrow} \mathcal{S}\left(\underline{C}^{n}, \underline{C}\right) \otimes \underline{C}^{n} \otimes \underline{C}^{n} \stackrel{\varepsilon_{\underline{C}^{n}, \underline{C}} \otimes \mathrm{id}}{\longrightarrow} \underline{C} \otimes \underline{C}^{n} \stackrel{\mathrm{id} \otimes \gamma_{n}}{\longrightarrow} \underline{C} \otimes \underline{C}^{\infty} \stackrel{\vec{\alpha}}{\longrightarrow} \underline{C} \\
& =\underline{\mathcal{S}}^{\mathrm{M}(C)}\left(\mathrm{A}(C)^{n}, \mathrm{~A}(C)\right) \otimes \underline{C}^{n} \stackrel{\mathrm{id} \otimes \gamma_{n}}{\longrightarrow} \underline{\mathcal{S}}^{\mathrm{M}(C)}\left(\mathrm{A}(C)^{n}, \mathrm{~A}(C)\right) \otimes \underline{C}^{\infty} \\
& \cong \underline{\mathcal{S}}^{\mathrm{M}(C)}\left(\mathrm{A}(C)^{n}, \mathrm{~A}(C)\right) \otimes I \otimes \underline{C}^{\infty} \stackrel{\mathrm{id} \otimes \eta_{n} \otimes \mathrm{id}}{\longrightarrow} \underline{\mathcal{S}}^{\mathrm{M}(C)}\left(\mathrm{A}(C)^{n}, \mathrm{~A}(C)\right) \otimes \underline{C}^{n} \otimes \underline{C}^{\infty} \\
& \stackrel{e_{n} \otimes \mathrm{id} \otimes \mathrm{id}}{\longrightarrow} \underline{\mathcal{S}}\left(\underline{C}^{n}, \underline{C}\right) \otimes \underline{C}^{n} \otimes \underline{C}^{\infty} \stackrel{\varepsilon_{\underline{C}^{n}, \underline{C}} \otimes \mathrm{id}}{\longrightarrow} \underline{C} \otimes \underline{C}^{\infty} \stackrel{\vec{\alpha}}{\longrightarrow} \underline{C} \\
& =\underline{\mathcal{S}}^{\mathrm{M}(C)}\left(\mathrm{A}(C)^{n}, \mathrm{~A}(C)\right) \otimes \underline{C}^{n} \stackrel{\mathrm{id} \otimes \gamma_{n}}{\longrightarrow} \underline{\mathcal{S}}^{\mathrm{M}(C)}\left(\mathrm{A}(C)^{n}, \mathrm{~A}(C)\right) \otimes \underline{C}^{\infty} \\
& \cong \underline{\mathcal{S}}^{\mathrm{M}(C)}\left(\mathrm{A}(C)^{n}, \mathrm{~A}(C)\right) \otimes I \otimes \underline{C}^{\infty} \stackrel{\mathrm{id} \otimes \eta_{n} \otimes \mathrm{id}}{\longrightarrow} \underline{\mathcal{S}}^{\mathrm{M}(C)}\left(\mathrm{A}(C)^{n}, \mathrm{~A}(C)\right) \otimes \underline{C}^{n} \otimes \underline{C}^{\infty} \\
& \stackrel{e_{n} \otimes \mathrm{id} \otimes \mathrm{id}}{\longrightarrow} \underline{\mathcal{S}}\left(\underline{C}^{n}, \underline{C}\right) \otimes \underline{C}^{n} \otimes \underline{C}^{\infty} \stackrel{\mathrm{id} \otimes\left\langle\pi_{i}^{n} \otimes \mathrm{id}\right\rangle_{i \in[n]}}{\longrightarrow} \underline{\mathcal{S}}\left(\underline{C}^{n}, \underline{C}\right) \otimes\left(\underline{C} \otimes \underline{C}^{\infty}\right)^{n} \\
& \stackrel{\operatorname{id} \times \vec{\alpha}^{n}}{\longrightarrow} \underline{\mathcal{S}}\left(\underline{C}^{n}, \underline{C}\right) \otimes \underline{C}^{n} \stackrel{\varepsilon_{\underline{C}^{n}, \underline{C}}}{\longrightarrow} \underline{C} \\
& =\underline{\mathcal{S}}^{\mathrm{M}(C)}\left(\mathrm{A}(C)^{n}, \mathrm{~A}(C)\right) \otimes \underline{C}^{n} \stackrel{e_{n} \otimes \mathrm{id}}{\longrightarrow} \underline{\mathcal{S}}\left(\underline{C}^{n}, \underline{C}\right) \otimes \underline{C}^{n} \stackrel{\operatorname{id} \otimes \gamma_{n}}{\longrightarrow} \underline{\mathcal{S}}\left(\underline{C}^{n}, \underline{C}\right) \otimes \underline{C}^{\infty} \\
& \cong \underline{\mathcal{S}}\left(\underline{C}^{n}, \underline{C}\right) \otimes I \otimes \underline{C}^{\infty} \stackrel{\operatorname{id} \otimes\left\langle\underline{\nu}_{i} \otimes \mathrm{id}\right\rangle_{i \in[n]}}{\longrightarrow} \underline{\mathcal{S}}\left(\underline{C}^{n}, \underline{C}\right) \otimes\left(\underline{C} \otimes \underline{C}^{\infty}\right)^{n} \\
& \stackrel{\operatorname{id} \otimes \vec{\alpha}^{n}}{\longrightarrow} \underline{\mathcal{S}}\left(\underline{C}^{n}, \underline{C}\right) \otimes \underline{C}^{n} \stackrel{\varepsilon_{\underline{C}^{n}, \underline{C}}}{\longrightarrow} \underline{C} \\
& =\underline{\mathcal{S}}^{\mathrm{M}(C)}\left(\mathrm{A}(C)^{n}, \mathrm{~A}(C)\right) \otimes \underline{C}^{n} \stackrel{e_{n} \otimes \mathrm{id}}{\longrightarrow} \underline{\mathcal{S}}\left(\underline{C}^{n}, \underline{C}\right) \otimes \underline{C}^{n} \stackrel{\operatorname{id} \otimes \gamma_{n}}{\longrightarrow} \underline{\mathcal{S}}\left(\underline{C}^{n}, \underline{C}\right) \otimes \underline{C}^{\infty} \\
& \stackrel{\operatorname{id} \otimes \kappa_{n}}{\longrightarrow} \underline{\mathcal{S}}\left(\underline{C}^{n}, \underline{C}\right) \otimes \underline{C}^{n} \stackrel{\varepsilon_{\underline{C}}^{n}, \underline{C}}{\longrightarrow} \underline{C} \\
& =\underline{\mathcal{S}}^{\mathrm{M}(C)}\left(\mathrm{A}(C)^{n}, \mathrm{~A}(C)\right) \otimes \underline{C}^{n} \stackrel{e_{n} \otimes \mathrm{id}}{\longrightarrow} \underline{\mathcal{S}}\left(\underline{C}^{n}, \underline{C}\right) \otimes \underline{C}^{n} \stackrel{\varepsilon_{\underline{C}^{n}, \underline{C}}}{\longrightarrow} \underline{C}
\end{aligned}
$$




\section{Acknowledgements.}

I am grateful to Marta Bunge, the editor of the present volume, and to the anonymous referees for comments that prompted further developments in the paper.

\section{References}

[1] T. Altenkirch, J. Chapman, and T. Uustalu. Monads need not be endofunctors. Logical Methods in Computer Science, 11(1:3):1-40, 2015.

[2] F. Borceux and B. Day. Universal algebra in a closed category. Journal of Pure and Applied Algebra, 16:133-147, 1980.

[3] P. M. Cohn. Universal Algebra. Harper's Series in Modern Mathematics. Harper \& Row, 1965.

[4] E. Dubuc. Enriched semantics-structure (meta) adjointness. Revista de la Unión Matemática Argentina, 25:5-26, 1970.

[5] M. Fiore, N. Gambino, M. Hyland, and G. Winskel. Relative pseudomonads, Kleisli bicategories, and substitution monoidal structures. Selecta Mathematica New Series, 2017.

[6] P. Freyd. Algebra valued functors in general and tensor products in particular. Colloquium Mathematicum, XIV:89-106, 1966.

[7] P. Freyd. Aspects of topoi. Bull. Austral. Math. Soc., 7:1-76, 1972.

[8] S. Lack and J. Rosický. Notions of Lawvere theory. Applied Categorical Structures, 19:363-391, 2011.

[9] F. W. Lawvere. Functorial semantics of algebraic theories and some algebraic problems in the context of functorial semantics of algebraic theories. Reprints in Theory and Applications of Categories, No. 5, pp 1-121. (Originally published as: Ph.D. thesis, Columbia University, 1963 and in Reports of the Midwest Category Seminar II, 1968, 41-61.).

[10] F. W. Lawvere. Metric spaces, generalized logic and closed categories. Reprints in Theory and Applications of Categories, No. 1, pp 1-37. (Originally published as: Rendiconti del Seminario Matematico e Fisico di Milano, XLIII (1973), 135-166.).

[11] R. Lucyshyn-Wright. Enriched algebraic theories and monads for a system of arities. Theory and Applications of Categories, 31(5):101-137, 2016.

[12] K. Menger. On substitutive algebra and its syntax. Mathematical Logic Quarterly, 10(6-7):81104, 1964.

[13] K. Nishizawa and A. J. Power. Lawvere theories enriched over a general base. Journal of Pure and Applied Algebra, 213(3):377-386, 2009.

[14] A. J. Power. Enriched Lawvere theories. Theory and Applications of Categories, 6(7):83-93, 1999. 
[15] A. J. Power. Discrete Lawvere theories. In Proceedings of the First International Conference on Algebra and Coalgebra in Computer Science, CALCO 2005, volume 3629 of Lecture Notes in Computer Science, pages 348-363, 2005.

[16] E. Redi. Representation of Menger systems by multiplace endomorphisms. Tartu Riikl. Ul. Toimetised (Uchen. Zapinski Tartu Gos. Univ.), 277:47-51, 1971. (Russian).

[17] A. A. L. Sangalli. On the structure and representation of clones. Algebra Universalis, 25:101$106,1988$.

[18] B. M. Schein and V. S. Trohimenko. Algebras of multiplace functions. Semigroup Forum, $17: 1-64,1979$.

[19] W. Taylor. A question on representing algebraic theories. Unpublished manuscript, 1979.

[20] W. Taylor. Abstract clone theory. In I. Rosenberg and G. Sabidussi, editors, Algebras and Orders, volume 389 of NATO ASI Series C: Mathematical and Physical Sciences, pages 507530, 1993. 